\title{
Analysis of Wide-Band Oscillation of Hybrid MMC Interfacing Weak AC Power System
}

\author{
Xiaojun Lu, Wang Xiang, Member, IEEE, Weixing Lin, Member, IEEE, Jinyu Wen, Member, IEEE
}

\begin{abstract}
The wide-band oscillation of the hybrid MMC induced by excessive power infeed under weak $\mathrm{AC}$ power system integration is analyzed in this paper. A closed-loop state-space-based time-domain small-signal model is firstly established to investigate the instability problem. Different from the findings in two-level VSCs or half-bridge MMCs, the root locus analysis and participation factor analysis in this paper reveals that the oscillation frequency and involved control loops are highly related to the operation status. When hybrid MMC operates as a rectifier, a low-frequency oscillation is observed with the $d$-channel control loop mainly participated. In contrast, a high-frequency oscillation occurs with a $q$-channel control loop mainly involved when the hybrid MMC operates as an inverter. This wide-band oscillation phenomenon is explored with the aid of two simplified loop-gain-based $s$-domain models, which are derived referring to the selective modal analysis approach. To suppress the oscillation, sensitivity analysis regarding the impact of parameters on the phase margin is conducted to recognize effective parameter adjustment methods. The analysis results are validated by detailed electromagnetic simulations.
\end{abstract}

Index Terms-Power system modeling, small-signal model, modular multilevel converter, weak AC system, HVDC transmission

\section{NOMENCLATURE}

\section{Subscripts}

$x, y$

axis of the rotating frame synchronized with AC system voltage

$d, q \quad$ axis of the rotating frame synchronized with the measured PCC voltage by PLL

pu per unit

fil filtered signals before entering the controller

ref reference values for the controller

0 steady-state values

$\begin{array}{ll}\text { Variables } & \\ u_{s} & \text { AC system voltage } \\ P & \text { AC-side active power injected into MMCs } \\ Q & \text { reactive power absorbed by MMCs } \\ u_{d c} & \text { DC terminal voltage of MMCs } \\ i_{d c} & \text { DC output current of MMCs }\end{array}$

This work is sponsored by the National Natural Science Foundation of China (51907068), the Joint Funds of the National Natural Science Foundation of China (U1766211), the China postdoctoral science foundation program (2018M642837), and the Key Research and Development Program of Shaanxi (2017ZDXM-GY-135). (Corresponding author: Wang Xiang)

$\mathrm{X}$. Lu, and J. Wen are with the State Key Laboratory of Advanced Electromagnetic Engineering and Technology, Huazhong University of Science and Technology, Wuhan 430074, China. (e-mail:luxiaojun1212@ foxmail.com, jinyu.wen @ hust.edu.cn).

W. Xiang is with the Department of Electronic and Electrical Engineering, University of Strathclyde, Glasgow, G1 1XW, U.K. (e-mail: xiangwang1003@foxmail.com).

W. Lin is with the TBEA SunOasis Co,. Ltd, Urumuchi 830011, Xinjiang, China. (e-mail: weixinglin@foxmail.com).

\author{
$i \quad$ AC-side current of MMCs \\ $i_{x}, i_{y} / i_{d}, i_{q} \quad$ axis components of $i$ under $x y / d q$ frame \\ $u_{p} \quad$ PCC voltage \\ $u_{p x}, u_{p y} / \quad$ axis components of $u_{p}$ under $x y / d q$ frame \\ $u_{p d}, u_{p q}$ \\ $i_{p}, i_{n}$ \\ $u_{c, a v g}$ \\ $M_{x}, M_{y}$ \\ $M_{d}, M_{q}$ \\ $M_{x 2}, M_{y 2} /$ \\ $M_{d 2}, M_{q 2}$ \\ $M_{d c}$ \\ $u_{p x 2}^{\Sigma}, u_{p y 2}^{\Sigma}$ \\ $u_{p x}^{\Sigma}, u_{p y}^{\Sigma}$ \\ $u_{p 0}^{\Sigma}$ \\ $i_{\text {diff }}$ \\ $i_{\text {diffo }}$ \\ $i_{\text {diff } x 2,} i_{\text {diffy } 2}$ \\ $e_{v}$ \\ $e_{d c}$ \\ $x_{P}, x_{Q}, x_{I d}, x_{I q}$ \\ $x_{P d c}, x_{I d c}$ \\ $\theta$ \\ upper and lower arm current \\ average capacitor voltage \\ fundamental-frequency modulation signal in $x y$ \\ / $d q$ frame \\ second harmonic modulation signal in $x y / d q$ \\ frame \\ DC modulation signal \\ second harmonic of the total capacitor voltage \\ in one phase arm under $x y$ frame \\ fundamental-frequency component of total \\ capacitor voltage in one arm under $x y$ frame \\ DC component of the total capacitor voltage \\ circulating current \\ DC component of $i_{\text {diff }}$ \\ second harmonic of $i_{\text {diff }}$ in $x y$ frame \\ AC-side internal output voltage of MMCs \\ DC-side internal output voltage of MMCs \\ state variables in ACC outer and inner loop \\ state variables in DCC outer and inner loop \\ PCC voltage phase angle
}

Parameters

$R_{S} / L_{s}$

$K_{T}$

$R_{T} / L_{T}$

$C_{s u b}$

$L_{\text {arm }} / R_{\text {arm }}$

$N_{\text {sub }}$

$K_{P O} / K_{P I}$

$K_{I O} / K_{I I}$

$L_{p u}$

$K_{P O d d} / K_{P I d c}$

$K_{I O d c} / K_{I I d c}$

$K_{\text {Ppll }} / K_{\text {Ipll }}$

$f_{p}, f_{\text {uac }}, f_{\text {iac }}$,

$f_{u c}, f_{u d c}, f_{i d c}$

equivalent $\mathrm{AC}$ system resistance/inductance turn-ratio of the $\mathrm{AC}$ transformer

$\mathrm{AC}$ transformer resistance/inductance capacitance of sub-modules in MMC inductance and equivalent resistance per arm total number of sub-modules in one MMC arm proportional gain for ACC outer/inner loop integral gain for $\mathrm{ACC}$ outer/inner loop feedforward gain for AC current in $\mathrm{ACC}$ proportional gain for DCC outer/inner loop integral gain for DCC outer/inner loop proportional and integral gain of PLL the cutting-off frequencies of the $2^{\text {nd }}$ order low-pass filters for power, AC voltage, AC current, average capacitor voltage, DC voltage and DC current.

\section{INTRODUCTION}

With the increasing impetus for bulk inland renewable power transmission, the overhead line (OHL) based modular multilevel converter high-voltage-direct-current (MMCHVDC) scheme is preferred, which puts forward high requirements for the DC fault current isolation. Till now, most of the MMC-HVDC projects adopt the half-bridge 
sub-modules (HBSMs) based topology, which cannot actively interrupt the DC fault current and needs the cooperation of high-power DC circuit breakers (DCCBs) [1][2]. The hybrid MMC consisting of HBSMs and full-bridge sub-modules (FBSMs) is promising in OHL-HVDC with its inherent ability to interrupt the DC fault current [3]. In the cable-based HVDC systems, the operation of the hybrid MMC can be the same as the half-bridge MMC during normal operation. When a DC fault is detected, the hybrid MMC will be blocked to interrupt the fault current. But for OHL application, due to the frequent occurrence of temporary DC faults, the operation of the hybrid MMC needs to be improved to facilitate DC-fault-ride-through without blocking the converters. Improved control strategies need to be proposed to realize the DC-fault-ride-through of the hybrid MMC. In a $\pm 800 \mathrm{kV}$ three-terminal OHL-HVDC project under construction in China [3], named as Kun-Liu-Long $(K L L)$ project, two terminals (Liubei and Longmen) adopt the hybrid MMC topology, and the decoupled $\mathrm{AC}$ and DC current control will be used [4]-[5].

Apart from the DC fault handling issue, the small-signal stability of the converters should also be investigated to avoid underlying oscillation problems, especially under weak AC system integration [6]-[7]. As reported, due to the interaction between the converter and the $\mathrm{AC}$ system, unexpected oscillations in the converters' current, voltage or power emerging from sub-synchronous frequency to high-frequency range are observed [7].

The stability problem for a half-bridge MMC project integrated with the weak AC system has occurred in China. The minimum short-circuit-ratio (SCR) of a $\pm 420 \mathrm{kV} /$ 1250MW back-to-back MMC-HVDC project in China can be as low as 1.9 [6], which imposes a limit to the transferable active power. A $1270 \mathrm{~Hz}$ resonance occurs in the $\mathrm{AC}$ voltages for a $\pm 350 \mathrm{kV} / 1000 \mathrm{MW}$ back-to-back MMC-HVDC project after AC transmission lines are out of service and SCR drops to 3.3 [8]. During the test of $K L L$ project, according to actual operation data, the SCR of the AC system connected to the Liubei terminal may drop below 2 due to the topology change caused either by AC fault clearance or scheduled transmission line maintenance. In this case, the Liubei terminal becomes connected to a weak AC system, which may impose stability risk for the KLL project. Therefore, both constructed and under-construction $\mathrm{MMC}$ projects are confronting the stability problem under a weak AC system connection. It is necessary to investigate the small-signal stability between MMCs and the weak AC system to guarantee a safe operation.

The stability between voltage-source-converters (VSCs) and the weak AC system has been an ever-lasting topic and attracted great interest both in industry and academia. The mainly researched topology till now includes two-level VSCs and half-bridge MMCs. In [9], the joint impact of SCR and phase-lock-loop (PLL) gain on the small-signal stability of the VSC under the active power and $\mathrm{AC}$ voltage control mode is investigated. While in [10] and [11], the mutual coupling between the power and $\mathrm{AC}$ voltage control loop is revealed under high active power penetration [10] or low SCR [11]. Besides the time-domain analysis, Wen et al proposed a $d q$ impedance model based on the measured frame for grid-tied
VSCs [12][13]. The negative resistance feature of $Z_{q q}$ is supposed to be the cause of the instability. Similarly in [14], $Z_{q q}$ is found to display negative resistance behavior within the PLL bandwidth. For the VSCs in wind farm applications, Huang and Wang et al proposed the small-signal model under the DC and AC bus voltage control mode[15][16]. While for half-bridge MMCs, the AC impedance model is derived in [8] and [17]. A low-pass filter is added for the feedforward point of common coupling (PCC) voltage, and the high-frequency resonance of the AC bus voltage is effectively eliminated [8]. The time-domain small-signal models for half-bridge MMCs are studied in [18]-[21]. Given that the control strategy of the hybrid MMC for OHL application is different from the traditional two-level VSCs or half-bridge MMCs, the small-signal model suitable for the hybrid MMCs remains to be investigated, and its stability characteristic under weak AC system integration remains to be explored.

Besides the absence of a suitable small-signal model for the hybrid MMC, some questions remain in the existing analytical models for VSCs. For example, the outer loop of the controller is neglected in [8], [12]-[14]. While the inner control loop is neglected in [15]-[16]. There is no agreement on which control dynamics should be ignored. Generally, the simplification is based on the different response speeds of the control loops corresponding to the special time scale, and the ignorance of a certain time scale consequently results in the exposure of oscillation in a limited frequency range. While for the wide-band oscillation problem, as addressed in this paper, various time scales should be considered, so the theoretical foundation needs to be established for the simplification of control dynamics, for example, based on the weighted contribution to the instability, rather than time-scales. The DC voltage dynamics should also be considered rather than regarded as constants such as in [9], [12]-[14], so the impact of the DC network or other terminals can be reflected. As revealed in [11] and [22], the impact of operation status on small-signal stability is remarkable, while most existing researches only focus on the inverter status. Both active power directions should be researched, especially for the master converter, the transmitted active power or power direction of which can be varied during operation.

The contributions of this paper are as follows:

1) The closed-loop state-space-based small-signal model for the hybrid MMC with fault-ride-through control strategy is derived for the first time.

2) A wide-band oscillation between the master hybrid MMC and the weak AC system is revealed by modal analysis and validated by simulation for the rectifier and inverter status.

3) The simplified $s$-domain models for oscillation analysis are derived referring to the selective modal analysis (SMA) approach [23], which produces satisfying results on judging the stability and oscillation frequency more efficiently compared with the complicated state-space small-signal model.

The paper is organized as follows. The time-domain small-signal model of the hybrid MMC is proposed and validated in Section II. Then, the wide-band oscillation under weak $\mathrm{AC}$ system integration for the inverter and rectifier 
operation is disclosed in simulation and analyzed by the small-signal model in Section III. Sensitivity studies for different parameters and the participation factor analysis for the wide-band oscillation are carried out in Section III. Due to the complexity of the time-domain model, the simplified $s$-domain models based on the SMA approach are studied in Section IV to analyze the stability more efficiently. Both time-domain and $s$-domain analysis have proposed proper parameter adjustment methods to enhance the stability, which is validated in Section V. Finally, the conclusion is drawn in Section VII.

\section{TIME-DOMAIN SMALL-SIGNAL MODEL}

\section{A. A brief introduction to the hybrid MMC}

Fig. 1 depicts the equivalent circuit of a hybrid MMC connected to the AC system through the AC transformer. Each arm of the hybrid MMC consists of HBSMs and FBSMs in series connection. All the variables are sampled and filtered before entering the control system. For OHL-based transmission, to avoid long-time power interruption, the hybrid MMC is required to ride through the DC faults without blocking in $K L L$ project.

The traditional direct current vector control for the half-bridge MMC is depicted in Fig. 2 (a). To facilitate DC fault-ride-through, an improved direct current vector control for hybrid MMC is designed in Fig. 2 (b) [4]-[5], which is also named as the AC/DC decoupled control strategy. The detailed control structure of the AC/DC decoupled control is depicted in Fig. 3, which will be implemented in the KLL project. The controller consists of the AC current control (ACC) loop, the DC current control (DCC) loop and the circulating current suppression control (CCSC) loop, which separately produces the fundamental modulation signal $M_{d} \cos (\omega t)+M_{q} \sin (\omega t)$, the DC modulation signal $M_{d c}$, and the second harmonic modulation signal $M_{d 2} \cos (2 \omega t)+M_{q 2} \sin (2 \omega t)$. The structures of ACC and CCSC are similar to those of the vector-based current controller for half-bridge MMCs. While the outer-loop control targets of the $d$-channel and $q$-channel for ACC are separately set to be $u_{c, \text { avg }}$ and $Q$. The outer-loop control target for DCC is $u_{d c}$ or $P$. The inner-loop of DCC regulates DC current.

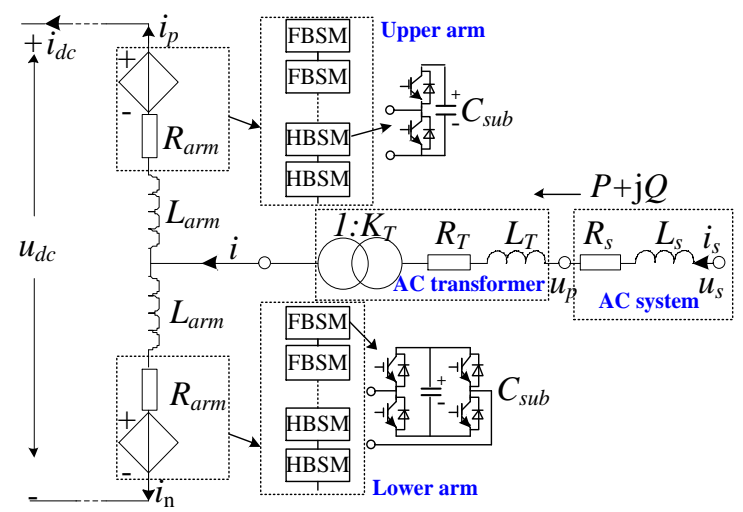

Fig. 1. Equivalent circuit of the hybrid MMC system for a single phase.

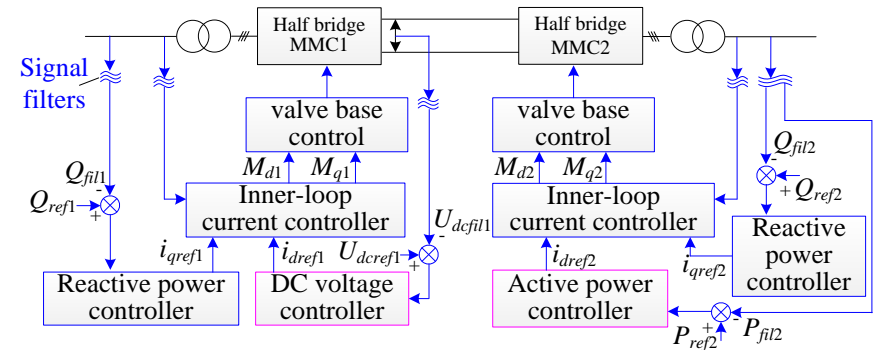

(a) traditional direct current vector control for the half-bridge MMC

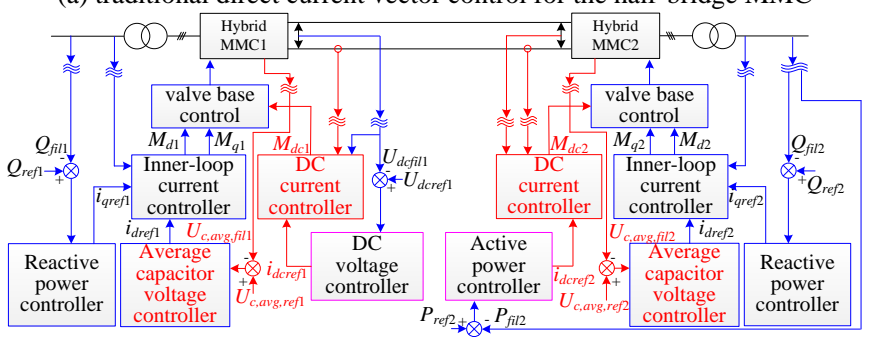

(b) improved direct current vector control for the hybrid MMC

Fig. 2. Direct current vector control for a point-to-point MMC system.

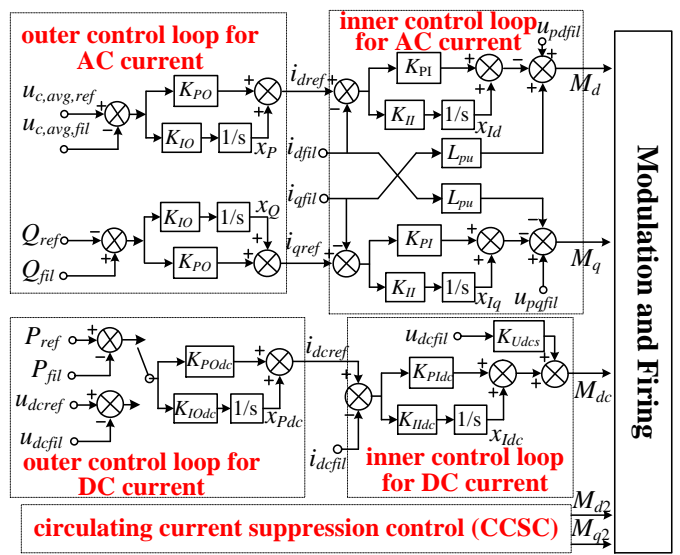

Fig. 3. AC/DC decoupled control strategy of the hybrid MMC.

\section{B. Small-signal model of the hybrid MMC}

The open-loop state-space model of the hybrid MMC is proposed in the authors' earlier work [26]. The state-space model is built under rotating frames using the dynamic phasor method for a single phase. The oscillating variables in the static $\mathrm{ABC}$ frame are transformed into the $x y$ rotating frame. The small-signal model can be written as:

$$
\Delta \dot{\boldsymbol{X}}_{8 t h}=\boldsymbol{A}_{8 t h} \Delta \boldsymbol{X}_{8 t h}+\boldsymbol{B}_{8 t h d c} \Delta u_{d c}+\boldsymbol{B}_{8 t h a c} \Delta \boldsymbol{I}_{a c}+\boldsymbol{B}_{8 t h c t r l} \Delta \boldsymbol{M}
$$

where $\boldsymbol{X}_{8 t h}=\left[u_{p x 2}^{\Sigma}, u_{p y 2}^{\Sigma}, u_{p x}^{\Sigma}, u_{p y}^{\Sigma}, u_{p 0}^{\Sigma}, i_{d i f f x 2}, i_{d i f f y 2}, i_{d i f f 0}\right]^{\mathrm{T}}$.

The state variables are described by the internal electric dynamics of the hybrid MMC. The input quantities include the AC current $\boldsymbol{I}_{a c}=\left[i_{x}, i_{y}\right]^{\mathrm{T}}$, the DC terminal voltage $u_{d c}$ and the modulation signals $\boldsymbol{M}=\left[\begin{array}{lllll}M_{x}, & M_{y}, & M_{x 2}, & M_{y 2}, & M_{d c}\end{array}\right]^{\mathrm{T}}$. The expressions for the matrices in (1) can be conveniently obtained from reference [26].

The electric system of the hybrid MMC outputs the AC internal voltage $\boldsymbol{e}_{v x y}=\left[e_{v x}, e_{v y}\right]^{\mathrm{T}}$ to the AC system, and outputs the DC internal voltage $e_{d c}$ and the DC current $i_{d c}=3 i_{d i f f 0}$ to the $\mathrm{DC}$ network. The equivalent circuit of the $\mathrm{AC}$ and $\mathrm{DC}$ port is depicted in Fig. 4.

The expressions for $e_{v}$ and $e_{d c}$ are: 


$$
\begin{aligned}
& {\left[\begin{array}{l}
e_{v x} \\
e_{v y}
\end{array}\right]=\left[\begin{array}{ccccc}
\frac{M_{x}}{4} & \frac{M_{y}}{4} & \frac{M_{x 2}-2 M_{d c}}{4} & \frac{M_{y 2}}{4} & \frac{M_{x}}{2} \\
\frac{-M_{y}}{4} & \frac{M_{x}}{4} & \frac{M_{y 2}}{4} & -\frac{M_{x 2}+2 M_{d c}}{4} & \frac{M_{y}}{2}
\end{array}\right] .} \\
& {\left[\begin{array}{lllll}
u_{p x 2}^{\Sigma} & u_{p y 2}^{\Sigma} & u_{p x}^{\Sigma} & u_{p y}^{\Sigma} & u_{p 0}^{\Sigma}
\end{array}\right]^{\mathrm{T}}} \\
& e_{d c}=M_{d c} u_{p 0}^{\Sigma}-\frac{1}{2}\left(M_{x} u_{p x}^{\Sigma}+M_{y} u_{p y}^{\Sigma}+M_{x 2} u_{p x 2}^{\Sigma}+M_{y 2} u_{p y 2}^{\Sigma}\right)
\end{aligned}
$$

In this paper, the control dynamics are added to form a closed-loop state-space-based small-signal model. Different from the electric dynamics, the control dynamics are modeled under the rotating frame synchronized with the measured PCC voltage by PLL, namely, $d q$ frame. The state-space model of

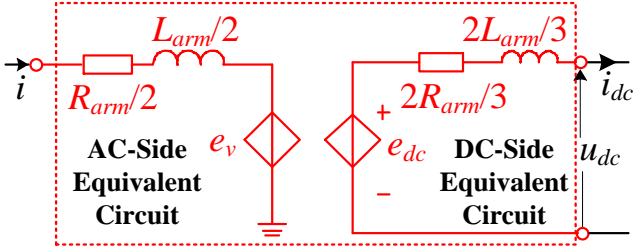

Fig. 4. Equivalent circuit of the hybrid MMC AC/DC ports.

the ACC, DCC, CCSC and PLL can be easily obtained by exploring the control block diagrams. The small-signal models of ACC and DCC are written as:

$$
\begin{gathered}
\Delta \dot{\boldsymbol{X}}_{C}=\boldsymbol{A}_{C} \Delta \boldsymbol{X}_{C}+\boldsymbol{B}_{C f i l} \Delta \boldsymbol{y}_{C f i l}+\boldsymbol{B}_{C r e f} \Delta \boldsymbol{u}_{C r e f} \\
\Delta \boldsymbol{M}_{d q}=\boldsymbol{C}_{C} \Delta \boldsymbol{X}_{C}+\boldsymbol{D}_{C f i l} \Delta \boldsymbol{y}_{C f i l}+\boldsymbol{D}_{C r e f} \Delta \boldsymbol{u}_{C r e f} \\
\Delta \dot{\boldsymbol{X}}_{C d c}=\boldsymbol{A}_{C d c} \boldsymbol{X}_{C d c}+\boldsymbol{B}_{C d c f i} \boldsymbol{y}_{C d c f i l}+\boldsymbol{B}_{C d c r e f} \boldsymbol{u}_{C d c r e f} \\
M_{d c}=\boldsymbol{C}_{C d c} \boldsymbol{X}_{C d c}+\boldsymbol{D}_{C d c f i} \boldsymbol{y}_{C d c f i l}+\boldsymbol{D}_{C d c r e f} \boldsymbol{u}_{C d c r e f}
\end{gathered}
$$

where $\boldsymbol{X}_{C}=\left[x_{P}, x_{Q}, x_{I d}, x_{I q}\right]^{\mathrm{T}}, \boldsymbol{u}_{C r e f}=\left[u_{c, a v g, r e f}, Q_{r e f}\right]^{\mathrm{T}}, \boldsymbol{y}_{C f i l}=\left[u_{c, a v g, f i l}\right.$, $\left.Q_{f i l}, i_{d f i l}, i_{q f i l}, u_{p d f i l}, u_{p q f i}\right]^{\mathrm{T}}, \boldsymbol{M}_{d q}=\left[M_{d}, M_{q}\right]^{\mathrm{T}}, \boldsymbol{X}_{C d c}=\left[x_{P d c}, x_{I d c}\right]^{\mathrm{T}}$, $\boldsymbol{u}_{\text {Cdcref }}=\left[P_{\text {ref }}, u_{d c r e f}, i_{d c r e f}\right]^{\mathrm{T}}, \boldsymbol{y}_{C d c f i}=\left[P_{\text {fil }}, u_{d c f i}, i_{d c f i l}\right]^{\mathrm{T}}$. The matrices in (4) and (5) can be directly derived from Fig. 3.

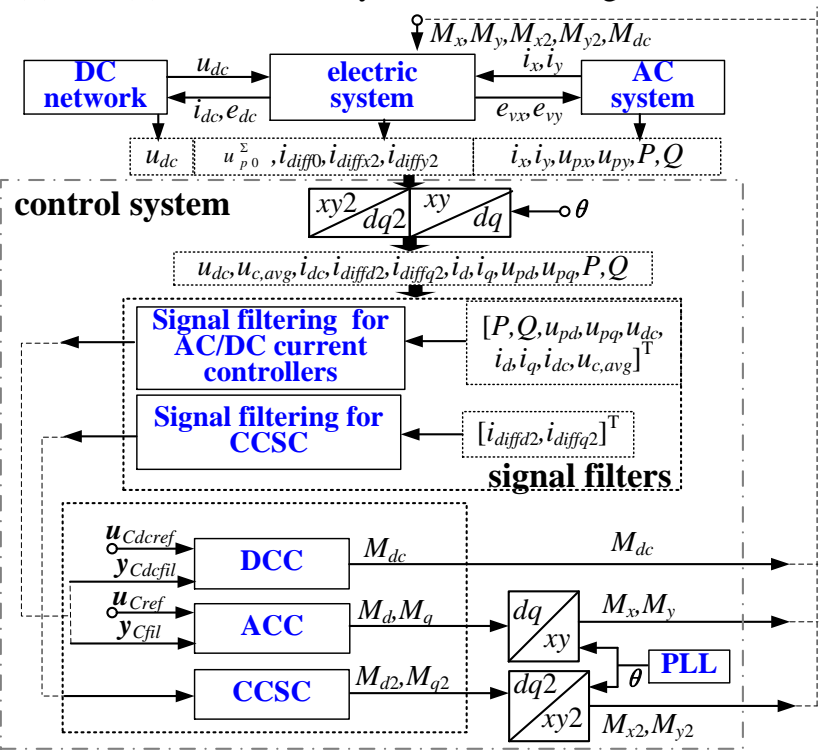

Fig. 5. Modeling frame for the closed-loop state-space model of hybrid MMC.

In this paper, the signal filters for controllers are modeled as the second-order low-pass filter. The small-signal model for CCSC, PLL and the signal filters can be referred to [21], which are the same as the models for half-bridge MMCs. The overall modeling frame for the closed-loop state-space model is depicted in Fig. 5. The overall small-signal model can be easily obtained by linearizing each of the subsystems in a modular way.

\section{Validation of the small-signal model}

In the following simulation, a two-terminal hybrid MMC system is built, as shown in Fig. 6. The DC line is equivalent to one $\pi$-section, which is also adopted in [27]-[29] for small-signal stability analysis.

The detailed parameters of the hybrid MMC and the AC system are displayed in the Appendix. The strength of the AC system connected to the hybrid MMC can be decided by SCR as calculated by:

$$
S C R=\frac{P_{a c}}{P_{d c N}}
$$

where $P_{a c}$ is the short-circuit capacity of the AC system, and $P_{d c N}$ is the rated capacity of the hybrid MMC. Since the AC system is equaled as a Thevenin circuit, $P_{a c}$ can be adjusted by varying the internal impedance $R_{s}+\mathrm{j} \omega L_{s}$. In this configuration, the SCRs of the AC system 1 and 2 are respectively 2 and 20, which indicates a weak and strong AC system.

The master hybrid MMC1 is connected to the weak AC system, which regulates the DC voltage and reactive power. While the other terminal hybrid MMC2 is connected to a strong AC grid, which regulates the active and reactive power. The electromagnetic model of the two-terminal system is built in PSCAD/EMTDC.

In order to distinguish the flexible control of the DC voltage, the reference for the DC voltage is intentionally set to be $70 \%$ of the rated value. The reactive power and active power are set to be 0.2 and 0.5 (in per unit). To validate the correctness of the proposed small-signal model, the DC voltage reference is decreased from 0.7 to 0.65 at $1 \mathrm{~s}$. The step responses of the electromagnetic model and the small-signal model are compared in Fig. 7, which shows good consistency.

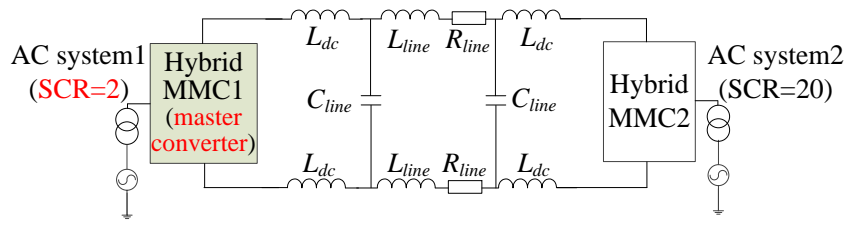

Fig. 6. The topology of the tested two-terminal hybrid MMC system.

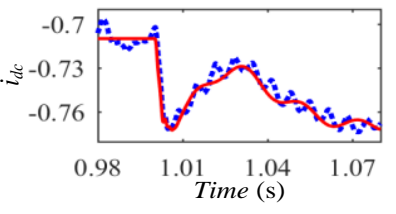

(a) DC current

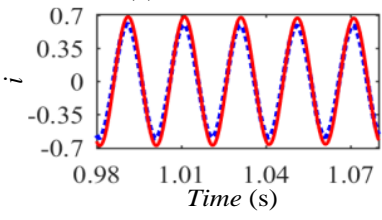

(c) AC current

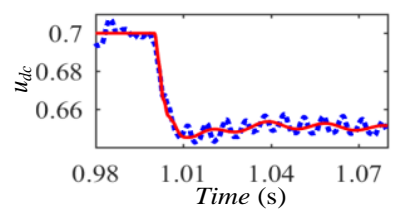

(b) DC voltage

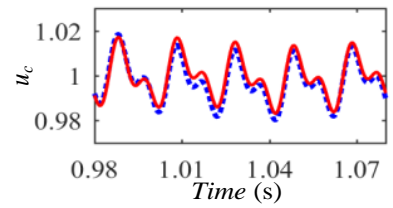

(d) total capacitor voltage 


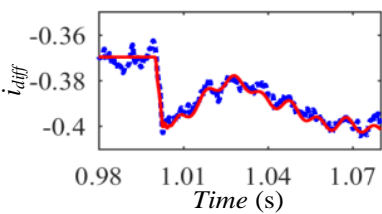

(e) circulating current

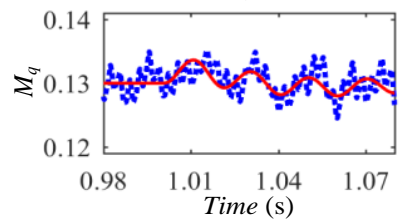

(g) ACC $q$-channel output

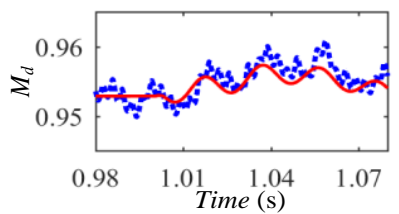

(f) ACC $d$-channel output

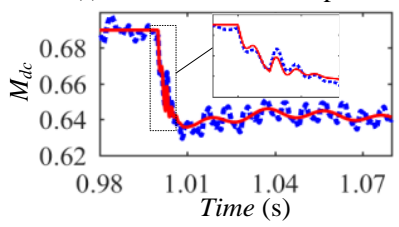

(h) DCC output
--.- PSCAD model — proposed small-signal model

Fig. 7. Validation of the small-signal model (all in per unit).

\section{Small-Signal Stability ANalysis Under Weak AC SYSTEM INTEGRATION}

Conventionally, the master hybrid MMC is connected to a strong AC system to support the DC voltage of the whole HVDC system and balance the active power. While under some scenarios, such as scheduled transmission line maintenance or incidental AC fault trip, the topology of the $\mathrm{AC}$ system varies, which may result in a sudden change of $\mathrm{AC}$ system parameters. Consequently, the hybrid MMC will face sudden SCR dip during operation while maintaining high-level active power transmission. This may lead to a wide-band oscillation problem, as will be disclosed in the simulation and explained by the small-signal stability analysis in this section.

\section{A. Simulation of the wide-band oscillation}

Suppose that the active power flowing from AC side to DC side is the default positive direction. In practice, the active power direction of the master converter may be bidirectional. In this paper, by adjusting the active power reference of hybrid MMC2, the operation status of hybrid MMC1 can be changed accordingly. When hybrid MMC1 is the receiving end, as shown in Fig. 8 (a), with the rise of the inverted active power, the active and reactive power start oscillating, while the average capacitor voltage remains stable. The dominant oscillation frequency at $P_{p u}=-0.56$ is $185.6 \mathrm{~Hz}$. When hybrid $\mathrm{MMC1}$ is the sending end, as shown in Fig. 8 (b), with the rise of the rectified active power, the average capacitor voltage and the active power start oscillating, while the reactive power contains slight oscillation. The dominant oscillation frequency at $P_{p u}=0.60$ is $7.33 \mathrm{~Hz}$.

Based on the simulation results, it is clear that for the tested hybrid MMC1 integration to a weak AC system with SCR below 2 , instability occurs when over $60 \%$ active power is transmitted. The frequency of the triggered oscillation in the active power, reactive power or the average capacitor voltage varies from several $\mathrm{Hz}$ to several hundred $\mathrm{Hz}$, showing wide-band oscillation characteristics. The active power transmission direction imposes a notable impact on the oscillation frequency or the electric variables with evident oscillation.
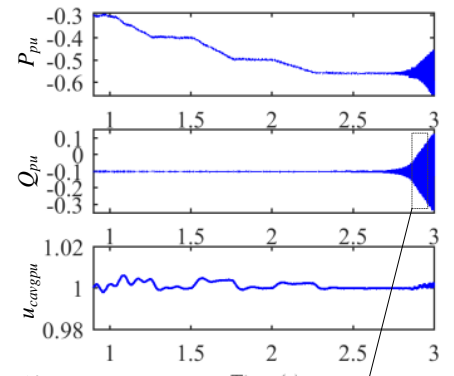

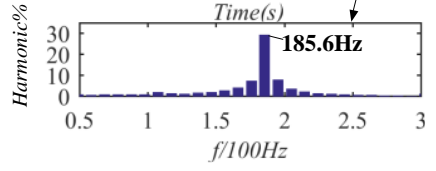

(a) inverter status

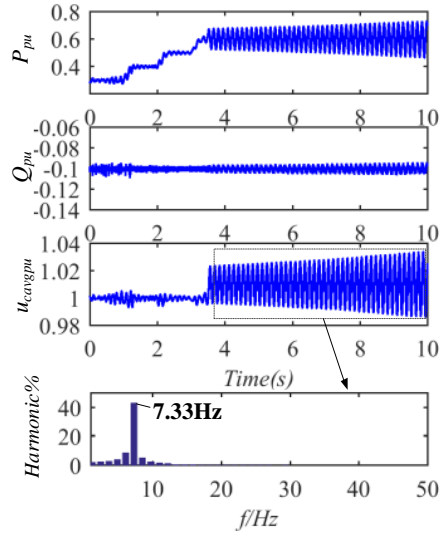

(b) rectifier status
Fig. 8. Instability caused by the high-level active power under weak AC system integration. Active power $\left(P_{p u}\right)$, reactive power $\left(Q_{p u}\right)$, average capacitor voltage $\left(u_{\text {cavgpu }}\right)$, harmonic component analysis.

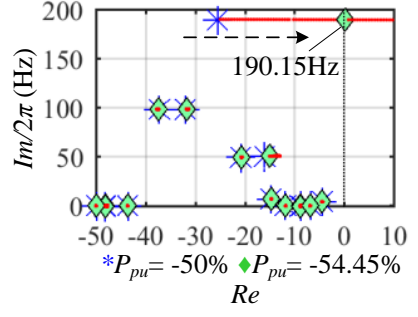

(a) inverter status

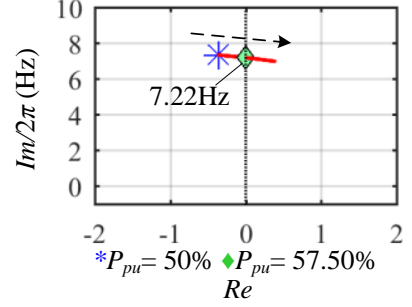

(b) rectifier status
Fig. 9. Root locus analysis with active power increasing.

\section{B. Root locus analysis}

Based on the small-signal model, the impact of the active power on small-signal stability is studied through root locus analysis. As shown in Fig. 9 (a), when the inverted active power exceeds $54.45 \%$ of the rated capacity, eigenvalues move from the left-half-plane (LHP) to the right-half-plane (RHP), which indicates an unstable system. Similarly, as shown in Fig. 9 (b), when the rectified active power exceeds $57.5 \%$ of the rated capacity, the system also becomes unstable.

Let the inverted active power be $56 \%$, and the RHP eigenvalues are calculated as $9.180 \pm \mathrm{j} 189.96 * 2 \pi$, which indicates that the oscillation frequency of the unstable mode is $189.96 \mathrm{~Hz}$. As can be seen, the calculated oscillation frequency is closed to the simulated oscillation frequency $185.6 \mathrm{~Hz}$ in Fig. 8 (a). Similarly, let the rectified active power be $60 \%$, and the RHP eigenvalues are calculated as $0.127 \pm \mathrm{j} 7.16 * 2 \pi$, which indicates $7.16 \mathrm{~Hz}$ oscillation frequency. The calculated oscillation frequency is also consistent with the simulated result $7.33 \mathrm{~Hz}$.

\section{Sensitivity analysis}

The sensitivity of the eigenvalue $\lambda_{\mathrm{i}}$ towards a certain parameter $\alpha$ can be calculated as $\Delta \lambda_{i} / \Delta \alpha$. The parameters to be analyzed include $Q$, PLL parameters $\left(K_{P p l l}, K_{\text {Ipll }}\right)$, ACC parameters $\left(K_{P O}, K_{I O}, K_{P I}, K_{I I}\right)$, DCC parameters $\left(K_{P O d c}, K_{I O d c}\right.$, $\left.K_{P I d c}, K_{I I d c}\right)$ and cutting-off frequencies of various signal filters $\left(f_{p}, f_{\text {uac }}, f_{\text {iac }}, f_{u c}, f_{\text {udc }}, f_{\text {idc }}\right)$.

The sensitivity calculation results for the real part of the critically unstable eigenvalue in Fig. 9 (a) and (b) are depicted in Fig. 10 (a) and (b) respectively. Under inverter status, the remarkable sensitivities are positive, which indicates that 
decreasing $K_{P O}, K_{P I}$ or the absorbed $Q$ can effectively pull the unstable mode to the LHP. While under rectifier status, the remarkable sensitivities are negative, which indicates that increasing $K_{P O}$ or the absorbed $Q$ can effectively pull the unstable mode to the LHP. Moreover, the remarkable sensitivities under inverter status are much larger than those under rectifier status, which indicates that the parameter adjustment is more effective under the inverter status with respect to enhancing the stability.

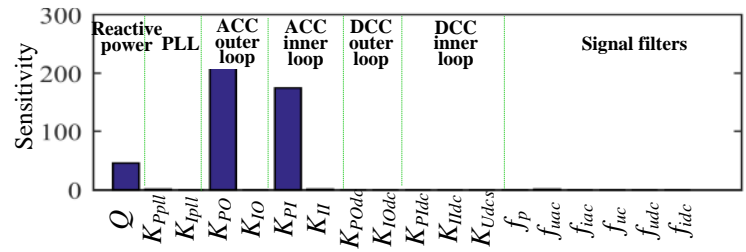

(a) inverter status

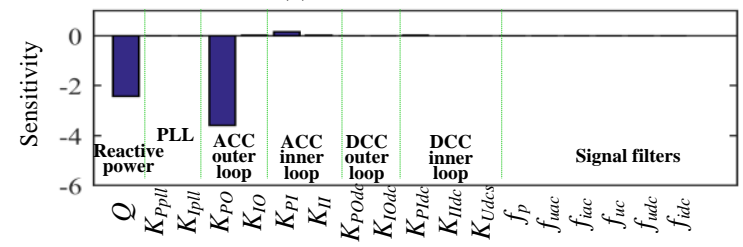

(b) rectifier status

Fig. 10. Sensitivity analysis of the critically unstable eigenvalue to parameters.

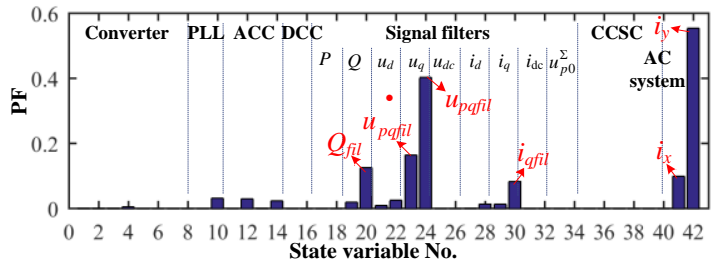

(a) inverter status

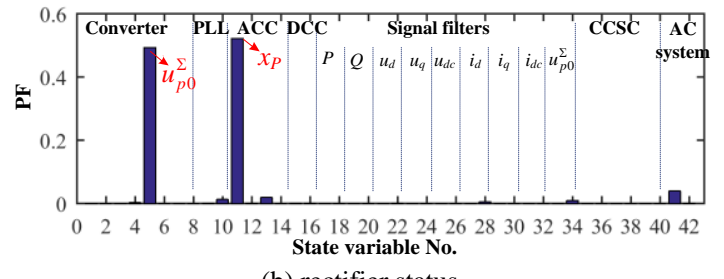

(b) rectifier status

Fig. 11. Participation factor analysis with critical active powers.

\section{D.Participation factor analysis}

Participation factor (PF) analysis is conducted to reveal the unstable modes under critical active power levels, as shown in Fig. 11. It is obvious that the distributions of PFs under two unstable modes are different to each other, which accords with the observation of the instability through different signals in Fig. 8.

As shown in Fig. 11 (a), the state variables with the two highest PFs are the measured reactive component of PCC voltage $u_{p q f i}$ and the reactive component of the AC current $i_{y}$. Since the unstable mode is concerned with the reactive-powerrelated state variables, it can be inferred that the small-signal stability of the inverted hybrid MMC is mainly affected by the control stability of ACC $q$-channel.

As shown in Fig. 11 (b), the unstable mode is mainly related to the average capacitor voltage and the outer loop state variable of ACC $d$-channel. Since the average capacitor voltage is the input signal of ACC $d$-channel, it can be inferred that the small-signal stability of the rectified hybrid MMC is mainly affected by the control stability of ACC $d$-channel.

\section{SIMPLIFIED S-DOMAIN MODELS}

The former participation factor analysis and simulation results reveal that the concerned wide-band oscillation is decoupled regarding the involved control loops, i.e., the ACC $q$-channel control mainly participates in the high-frequency oscillation under inverter status and the ACC $d$-channel control is related to the low-frequency oscillation under rectifier status. In this section, we refer to the selective modal analysis approach and simplify the analysis model based on loop-gain, in order to obtain a more intuitive understanding of the instability.

\section{A. SMA approach}

SMA is firstly presented in [23]. Originally, this analysis approach is used to propose a reduced-order model that accurately contains the eigen-structure of the selected modes of interest [24][25]. The first step of SMA is to classify the system state variables into relevant state variables (RSVs) and less relevant state variables (LRSVs) by participation factor analysis to the selected modes. Then, the dynamic models for LRSV are converted to static models by replacing the 's' Laplace operators with eigenvalues of the selected modes. In this way, the system is reduced into a condensed form for analyzing the selected modes without changing the eigen-structure. For more information about SMA, please refer to [23].

In SMA approach, the dynamics of LRSVs are neglected in the state-space-based reduced order model. In this paper, we refer to this idea and neglect the small-signal expressions of the distinguished LRSVs, in order to effectively derive a simplified loop-gain-based model.

\section{B. Simplified s-domain model of ACC q-channel under inverter status}

Based on Fig. 11 (a), the RSVs to the unstable mode come from the AC current, PLL, ACC $q$-channel and signal filters for $Q, u_{p q}$ and $i_{q}$.

The complete small-signal model for $i_{y}$ of the hybrid MMC can be condensed by neglecting the LRSVs, as shown below. Notice that the control inputs $\Delta M_{x}$ and $\Delta M_{y}$ should be retained.

$$
\begin{aligned}
s \Delta i_{y}= & \frac{1}{4 L_{t o t}}\left[M_{y 0} \Delta u_{p x 2}^{\Sigma}+\left(M_{x 20}+2 M_{d c 0}\right) \Delta u_{p y}^{\Sigma}-\right. \\
& 2 M_{y 0} \Delta u_{p 0}^{\Sigma}-M_{x 0} \Delta u_{p y 2}^{\Sigma}-\left(u_{p x 20}^{\Sigma}-2 u_{p 00}^{\Sigma}\right) \Delta M_{y}- \\
& M_{y 20} \Delta u_{p x}^{\Sigma}+u_{p y 20}^{\Sigma} \Delta M_{x}+u_{p y 0}^{\Sigma} \Delta M_{x 2}-u_{p x 0}^{\Sigma} \Delta M_{y 2}+ \\
& \left.2 u_{p y 0}^{\Sigma} \Delta M_{d c}-4 \omega L_{t o t} \Delta i_{x}-4 R_{t o t} \Delta i_{y}\right]
\end{aligned}
$$

where $L_{\text {tot }}=L_{\text {arm }} / 2+\left(L_{T}+L_{s}\right) / K_{T}^{2}, R_{t o t}=R_{\text {arm }} / 2+\left(R_{T}+R_{s}\right) / K_{T}^{2}$.

Based on (7), we can express $\Delta i_{y}$ with $\Delta M_{x}$ and $\Delta M_{y}$ as:

$$
\Delta i_{y} \approx-\frac{u_{p y 20}^{\Sigma}}{4\left(s L_{t o t}+R_{t o t}\right)} \Delta M_{x}+\frac{u_{p x 20}^{\Sigma}-2 u_{p 00}^{\Sigma}}{4\left(s L_{t o t}+R_{t o t}\right)} \Delta M_{y}
$$


Though the participation factor of $\Delta i_{x}$ in Fig. 11 (a) is not zero, we assume that $i_{x}$ is not closely related to ACC $q$-channel control. Thus, we neglect $\Delta i_{x}$ in order to simplify the derivation for the open-loop gain. In this way, the small-signal models of PCC voltage can be approximated by ignoring $\Delta i_{x}$ :

$$
\begin{gathered}
\Delta u_{p x}=\frac{\omega L_{s}}{K_{T}} \Delta i_{y}-\left(\frac{R_{s}}{K_{T}}+\frac{s L_{s}}{K_{T}}\right) \Delta i_{x} \\
\Delta u_{p y}=-\frac{\omega L_{s}}{K_{T}} \Delta i_{x}-\left(\frac{R_{s}}{K_{T}}+\frac{s L_{s}}{K_{T}}\right) \Delta i_{y}
\end{gathered}
$$

The small-signal model of the PLL can be expressed as:

$$
\Delta \theta=\left[\frac{K_{P p l l} s+K_{I p l l}}{s^{2}+K_{P p l l} s+K_{I p l l}}\right]_{G_{P L L}} \frac{u_{p x 0} \Delta u_{p y}-u_{p y 0} \Delta u_{p x}}{u_{p x 0}^{2}+u_{p y 0}^{2}}
$$

Substitute (9) and (10) into (11), and we can obtain the relation between $\Delta \theta$ and $\Delta i_{y}$ :

$$
\Delta \theta \approx\left[G_{P L L} \frac{-s L_{s} u_{p x 0}-R_{s} u_{p x 0}-\omega L_{s} u_{p y 0}}{K_{T}\left(u_{p x 0}^{2}+u_{p y 0}^{2}\right)}\right]_{G_{\theta_{-} y}} \Delta i_{y}
$$

The relation of $x y$ frame and $d q$ frame can be expressed as:

$$
\begin{aligned}
{\left[\begin{array}{l}
\Delta \beta_{d} \\
\Delta \beta_{q}
\end{array}\right] } & =\left[\begin{array}{cc}
\cos \theta_{0} & \sin \theta_{0} \\
-\sin \theta_{0} & \cos \theta_{0}
\end{array}\right]\left[\begin{array}{l}
\Delta \beta_{x} \\
\Delta \beta_{y}
\end{array}\right]+ \\
& {\left[\begin{array}{cc}
-\sin \theta_{0} & \cos \theta_{0} \\
-\cos \theta_{0} & -\sin \theta_{0}
\end{array}\right]\left[\begin{array}{l}
\beta_{x 0} \\
\beta_{y 0}
\end{array}\right] \Delta \theta }
\end{aligned}
$$

The ACC $d$-channel output $\Delta M_{d}$ is omitted for ACC $q$-channel analysis, then $\Delta M_{x}$ and $\Delta M_{y}$ can be approximated:

$$
\begin{gathered}
\Delta M_{x}=\cos \theta_{0} \Delta M_{d}-\sin \theta_{0} \Delta M_{q}+ \\
\quad\left[-\left(M_{d 0} \sin \theta_{0}+M_{q 0} \cos \theta_{0}\right)\right]_{G_{M x_{-} \theta}} \Delta \theta \\
\Delta M_{y}=\sin \theta_{0} \Delta M_{d}+\cos \theta_{0} \Delta M_{q}+ \\
\quad\left(M_{d 0} \cos \theta_{0}-M_{q 0} \sin \theta_{0}\right)_{G_{M y_{-} \theta}} \Delta \theta
\end{gathered}
$$

Substituting (14) and (15) into (8), we can obtain:

$$
\begin{gathered}
\Delta i_{y} \approx\left[\frac{\sin \theta_{0} u_{p y 20}^{\Sigma}+\cos \theta_{0}\left(u_{p x 20}^{\Sigma}-2 u_{p 00}^{\Sigma}\right)}{4\left(s L_{t o t}+R_{t o t}\right)}\right]_{G_{i y_{-} M q}} \Delta M_{q}+ \\
{\left[\frac{-G_{M x_{-} \theta} u_{p y 20}^{\Sigma}+G_{M y_{-} \theta}\left(u_{p x 20}^{\Sigma}-2 u_{p 00}^{\Sigma}\right)}{4\left(s L_{t o t}+R_{t o t}\right)}\right]_{G_{i y_{-} \theta}} \Delta \theta}
\end{gathered}
$$

Substitute (12) into (16), and $\Delta i_{y}$ can be expressed by $\Delta M_{q}$. In the following derivation, we shall express $\Delta M_{q}$ with $\Delta i_{y}$ by considering ACC $q$-channel dynamics.

By neglecting the LRSV $\Delta i_{d f i l}$, the small-signal model of the output of ACC $q$-channel is approximated as:

$$
\Delta M_{q}=\Delta u_{p q f i l}+G_{i n} \Delta i_{q f i l}+G_{i n} G_{\text {out }}\left(\Delta Q_{r e f}-\Delta Q_{f i l}\right)-L_{p u} \Delta i_{\text {dfil }}
$$

where $G_{\text {in }}$ and $G_{\text {out }}$ are respectively the transfer functions of the inner and outer loop of ACC.

$$
G_{\text {in }}(s)=K_{P I}+\frac{K_{I I}}{s}, G_{\text {out }}(s)=K_{P O}+\frac{K_{I O}}{s}
$$

The complete calculation for $\Delta Q$ is:

$$
\Delta Q=\frac{3}{2 K_{T}}\left(u_{p y 0} \Delta i_{x}-u_{p x 0} \Delta i_{y}+i_{x 0} \Delta u_{p y}+i_{y 0} \Delta u_{p x}\right)
$$

By substituting (9)-(10) into (19) and neglecting $\Delta i_{x}$, we can approximate the relation between $\Delta Q$ and $\Delta i_{y}$ :

$$
\Delta Q \approx\left[-s \frac{3 L_{s} i_{x 0}}{2 K_{T}^{2}}-\frac{3}{2 K_{T}}\left(u_{p x 0}+\frac{R_{s} i_{x 0}}{K_{T}}+\frac{\omega L_{s} i_{y 0}}{K_{T}}\right)\right]_{G_{Q_{-} i y}} \Delta i_{y}
$$

$\Delta i_{q}$ can be approximated by neglecting $\Delta i_{x}$ :

$$
\begin{aligned}
& \Delta i_{q}=\cos \theta_{0} \Delta i_{y}-\sin \theta_{0} \Delta i_{x}+ \\
& \quad\left(-i_{x 0} \cos \theta_{0}-i_{y 0} \sin \theta_{0}\right)_{G_{i_{q_{-}} \theta}} \Delta \theta
\end{aligned}
$$

Substitute (12) into (21), and we can express $\Delta i_{q}$ with $\Delta i_{y}$ :

$$
\Delta i_{q} \approx\left(\cos \theta_{0}+G_{i q_{-} \theta} G_{\theta_{-} i y}\right)_{G_{i q_{-} i y}} \Delta i_{y}
$$

$\Delta u_{p q}$ is approximated by substituting (9) and (10) into (23):

$$
\begin{gathered}
\Delta u_{p q}=\cos \theta_{0} \Delta u_{p y}-\sin \theta_{0} \Delta u_{p x}+ \\
\left(-\cos \theta_{0} u_{p x 0}-\sin \theta_{0} u_{p y 0}\right)_{G_{u q_{-} \theta}} \Delta \theta \\
\Delta u_{p q} \approx\left(G_{u q_{-} \theta} G_{\theta_{-i y}}-\frac{\cos \theta_{0} L_{s} s+\cos \theta_{0} R_{s}+\sin \theta_{0} \omega L_{s}}{K_{T}}\right)_{G_{u q_{-} i y}} \Delta i_{y}
\end{gathered}
$$

Suppose that transfer functions of signal filters for the AC current, AC voltage and the reactive power are respectively $G_{f i a c}, G_{f u a c}$ and $G_{f p}$, then, the variables on the right side of (17) can all be expressed by $\Delta i_{y} . \Delta M_{q}$ can be expressed as:

$$
\begin{gathered}
\Delta M_{q} \approx\left(G_{\text {uq_iy }} G_{\text {fuac }}+G_{i n} G_{i q_{-} i y} G_{f i a c}\right)_{G_{M q_{-} i y}} \Delta i_{y}+ \\
G_{\text {in }} G_{\text {out }}\left(\Delta Q_{\text {ref }}-\Delta Q_{\text {fil }}\right)
\end{gathered}
$$

Based on the derivation above, the block diagram for ACC $q$-channel control model is shown in Fig. 12. The open-loop transfer function can be obtained as:

$$
G_{A C C q}(s)=\frac{G_{i n} G_{o u t} G_{i y_{\_} M q} G_{Q_{\_} i y} G_{f p}}{1-G_{i y_{-} \theta} G_{\theta_{-} i y}-G_{i y_{-} M q} G_{M q_{-} i y}}
$$

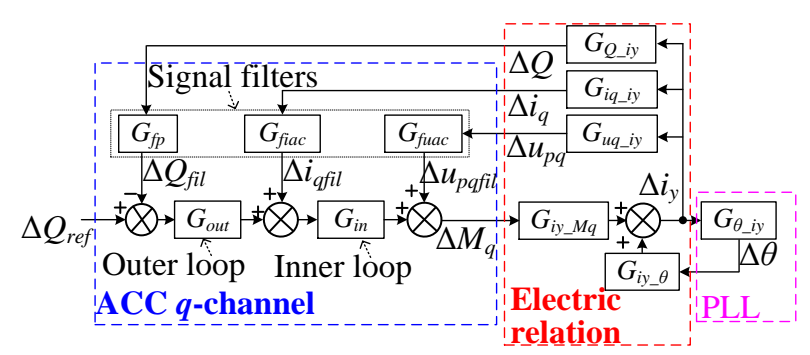

Fig. 12. Transfer function diagram of ACC $q$-channel control for analyzing the instability.

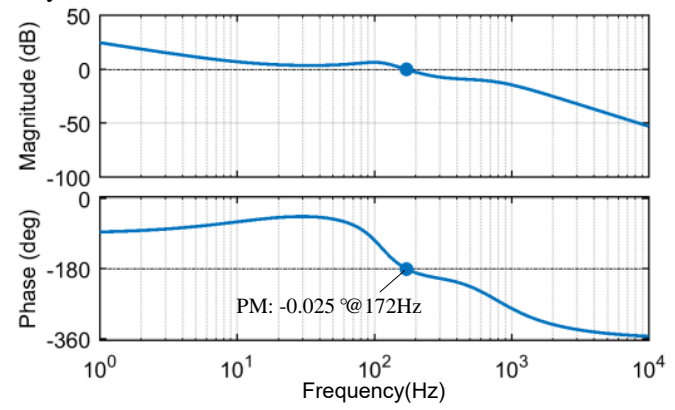

Fig. 13. Bode diagram of $G_{A C C q}$ under $54.45 \%$ inverted active power. 


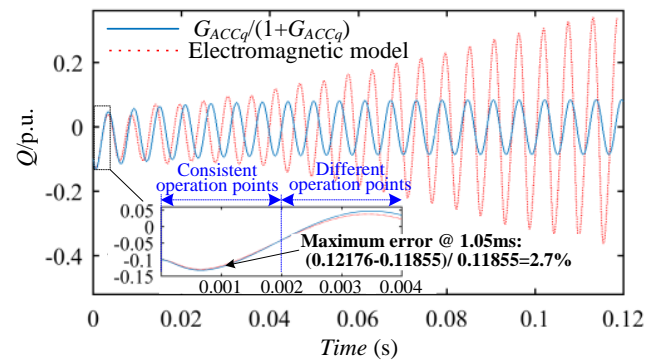

(a) step response of the reactive power

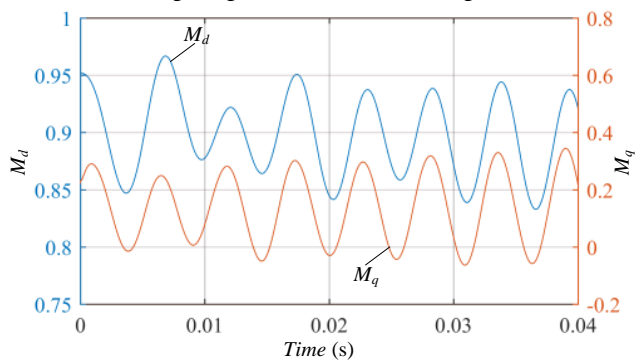

(b) outputs of ACC $d$ and $q$ channel in electromagnetic model

Fig. 14. Step response comparison of the reactive power between the simplified model and electromagnetic model.

\section{Accuracy verification and analysis of $G_{A C C q}(s)$}

Let the inverted active power of the hybrid MMC be the critical value 0.5445 p.u., and the corresponding bode diagrams of $G_{A C C q}$ are presented in Fig. 13.

As shown in Fig. 13, the phase margin (PM) of $G_{A C C q}$ is narrowly negative $\left(-0.0025^{\circ}\right)$, which indicates a critically unstable state. The crossover frequency is $172.2 \mathrm{~Hz}$, which is a little deviated from the mode calculation result $190 \mathrm{~Hz}$ or the electromagnetic simulation results $185.6 \mathrm{~Hz}$.

To explain this error, the closed-loop step response of $G_{A C C q} /\left(1+G_{A C C q}\right)$ is compared with the electromagnetic model. As shown in Fig. 14 (a), the reactive power is stepped from -0.1 p.u. to zero. The first swings of the two oscillation waveforms match relatively well. Considering that the operation points of the two models can be regarded consistent within $2 \mathrm{~ms}$, the maximum error of the two waveforms within $2 \mathrm{~ms}$ is calculated, which is only $2.7 \%$. However, the error becomes larger with the increase of time, showing different oscillation frequencies. This is caused by the variation of the operation points. The simplified model $G_{A C C q}$ is derived based on certain operation point, and neglects the impacts of other current control loops. However, as shown in Fig. 14 (b), the oscillation in the output of ACC $d$-channel $M_{d}$ gradually increases, which consequently affects the operation point. Within $2 \mathrm{~ms}$, the variation of $M_{d}$ is below 5\%, and its impact on the operation point can be neglected, thus it is reasonable to only calculate the maximum error within $2 \mathrm{~ms}$ in the step response.

Nevertheless, $G_{A C C q}$ can also be employed to analyze the impact of various parameters on stability by comparing PMs under different configurations. We assume that the variation of certain parameter will not change the classification of RSVs and LRSVs regarding the concerned mode. This is actually guaranteed through detailed calculation of PFs under different configurations. As shown in Fig. 15 (a) and (b), even though $f_{p}$ and $f_{\text {uac }}$ are varied at a wide range, the classification of RSVs and LRSVs almost keeps unchanged.

TABLE I displays the PM variations against different parameters. The increments of PM are different. PM can be obviously increased by reducing the proportion gains for ACC, or by reducing cutting-off frequencies for power and $\mathrm{AC}$ voltage signal filters. In contrast, increasing the output reactive power or reducing PLL gain cannot significantly increase PM.

To highlight the effective parameter adjustment methods, Fig. 16 depicts the corresponding bode plots. As shown in Fig. 16 (a), PM rises up to $38.9^{\circ}$ after $K_{P O}$ decreases from 1.0 to 0.5 . Similarly, PM rises up to $13.8^{\circ}$ after $K_{P I}$ decreases from 1.0 to 0.5 in Fig. 16 (b). The impact of control parameters on the stability accords with the sensitivity analysis results in Fig. 10 (a). It should be noticed that the reduction of $K_{P O}$ or $K_{P I}$ indicates the reduction of control bandwidth of the ACC $q$-channel outer or inner control loop, leading to the deterioration of reactive power and reactive current control.

While in Fig. 16 (c), with the reduction of $f_{p}$ for the reactive power, the PM decreases at first and then increases with a further reduction of $f_{p}$. In this case, the PM equals to $37.8^{\circ}$ when $f_{p}=40 \mathrm{~Hz}$. In Fig. 16 (d), the PM keeps increasing with the reduction of $f_{\text {uac }}$. Notice that the sensitivities for the cutting-off frequencies of the signal filters in Fig. 10 are not notable. This is due to the difference in the considered variation range of the cutting-off frequencies for the calculation. For the sensitivity study in Fig. 10, the variation step is set to be only $0.1 \mathrm{~Hz}$ away from the default values.

Based on the analysis above, it is recommended to slow down the ACC $q$-channel control or to deliberately decrease the cutting-off frequency of the signal filters for the reactive power signal or PCC voltage signal to enhance the stability.

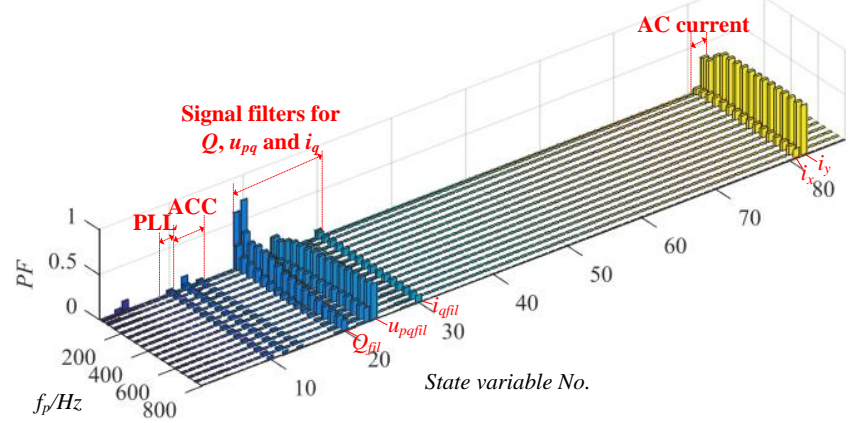

(a) variation of participation factors with changing $f_{p}$

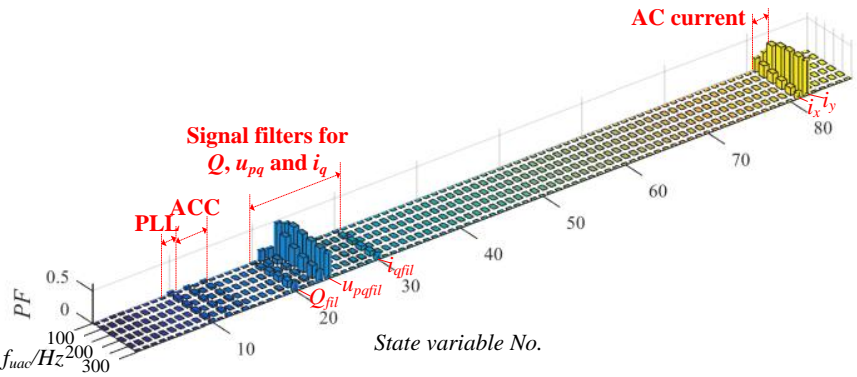

(b) variation of participation factors with changing $f_{\text {uac }}$

Fig. 15. Variation of participation factors with changing cutting-off frequencies of the signal filters at a wide range. 
TABLE I

Phase MARgin VARIATION AGAINST DifFERENT PARAMETERS UNDER INVERTER OPERATION

\begin{tabular}{llll}
\hline \hline Parameters & & Value variation & Phase margin variation \\
\hline \multirow{4}{*}{ ACC } & $K_{P O}$ & $1 \rightarrow 0.5$ & $-0.025^{\circ} \rightarrow 38.9^{\circ}$ \\
& $K_{I O}$ & $50 \rightarrow 10$ & $-0.025^{\circ} \rightarrow 3.89^{\circ}$ \\
& $K_{P I}$ & $1 \rightarrow 0.5$ & $-0.025^{\circ} \rightarrow 13.8^{\circ}$ \\
& $K_{I I}$ & $100 \rightarrow 20$ & $-0.025^{\circ} \rightarrow 4.37^{\circ}$ \\
\hline \multirow{3}{*}{ Signal filters } & $f_{p}$ & $800 \mathrm{~Hz} \rightarrow 40 \mathrm{~Hz}$ & $-0.025^{\circ} \rightarrow 37.8^{\circ}$ \\
& $f_{\text {uac }}$ & $300 \mathrm{~Hz} \rightarrow 50 \mathrm{~Hz}$ & $-0.025^{\circ} \rightarrow 72.2^{\circ}$ \\
& $f_{\text {iac }}$ & $800 \mathrm{~Hz} \rightarrow 2000 \mathrm{~Hz}$ & $-0.025^{\circ} \rightarrow 8.52^{\circ}$ \\
\hline PLL & $K_{P p l l}$ & $80 \rightarrow 1$ & $-0.025^{\circ} \rightarrow 5.74^{\circ}$ \\
\hline Reactive power & $Q$ & $-10 \% \rightarrow-40 \%$ (p.u.) & $-0.025^{\circ} \rightarrow 0.567^{\circ}$ \\
\hline \hline
\end{tabular}

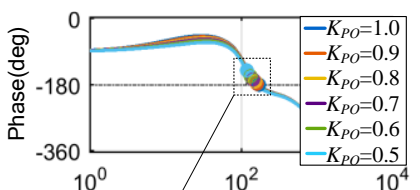

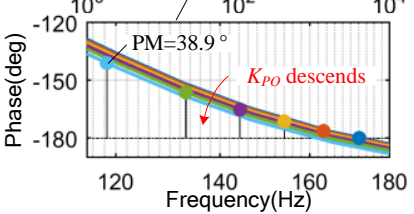

(a) decreasing $K_{P O}$

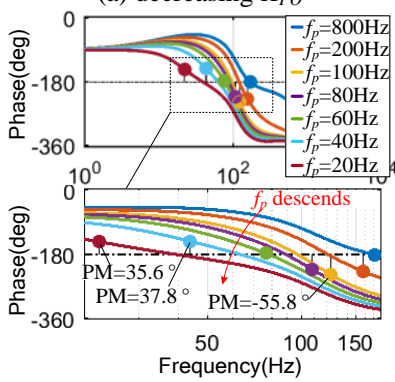

(c) decreasing $f_{p}$

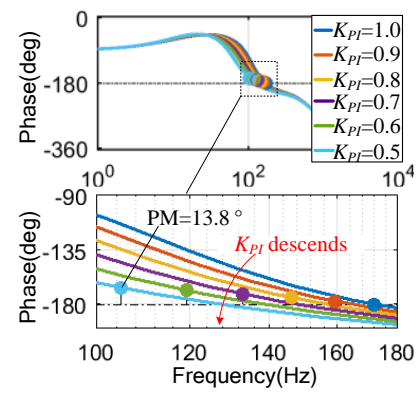

(b) decreasing $K_{P I}$

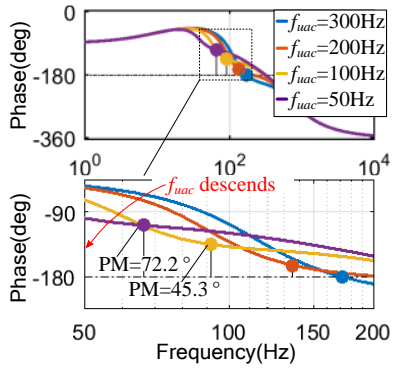

(d) decreasing $f_{\text {uac }}$
Fig. 16. Effective parameter adjustment methods to increase phase margins.

D.Simplified s-domain model for ACC d-channel under rectifier status

Based on Fig. 11 (b), the RSVs to the unstable mode are related to $u_{p 0}^{\Sigma}$, ACC $d$-channel, AC current $\left(\Delta i_{x}\right)$ and PLL.

The complete expression for $\Delta u_{p 0}^{\Sigma}$ can be condensed by neglecting LRSVs and retaining $\Delta M_{x}$ and $\Delta M_{y}$ :

$$
\begin{aligned}
& s \Delta u_{p 0}^{\Sigma}=\frac{1}{8 C_{a r m}}\left[-4 M_{d c 0} \Delta i_{d i f f 0}+2 M_{x 20} \Delta i_{d i f f x 2}+\right. \\
& 2 M_{y 20} \Delta i_{d i f f y 2}+M_{x 0} \Delta i_{x}+M_{y 0} \Delta i_{y}-4 i_{d i f f 00} \Delta M_{d c}+ \\
& \left.i_{x 0} \Delta M_{x}+i_{y 0} \Delta M_{y}+2 i_{d i f f x 20} \Delta M_{x 2}+2 i_{d i f f y 20} \Delta M_{y 2}\right]
\end{aligned}
$$

Then, $\Delta u_{p 0}^{\Sigma}$ can be approximated as:

$$
\Delta u_{p 0}^{\Sigma} \approx \frac{M_{x 0}}{8 C_{a r m} s} \Delta i_{x}+\frac{i_{x 0}}{8 C_{a r m} s} \Delta M_{x}+\frac{i_{y 0}}{8 C_{a r m} s} \Delta M_{y}
$$

The complete expression for $\Delta i_{x}$ can be condensed as:

$$
\begin{aligned}
s \Delta i_{x}= & \frac{1}{4 L_{t o t}}\left[-M_{x 0} \Delta u_{p x 2}^{\Sigma}-M_{y 0} \Delta u_{p y 2}^{\Sigma}-M_{y 20} \Delta u_{p y}^{\Sigma}+\right. \\
& \left(2 M_{d c 0}-M_{x 20}\right) \Delta u_{p x}^{\Sigma}-2 M_{x 0} \Delta u_{p 0}^{\Sigma}-4 R_{t o t} \Delta i_{x}+ \\
& 4 \omega L_{t o t} \Delta i_{y}-\left(u_{p x 20}^{\Sigma}+2 u_{p 00}^{\Sigma}\right) \Delta M_{x}-u_{p y 20}^{\Sigma} \Delta M_{y}- \\
& \left.u_{p x 0}^{\Sigma} \Delta M_{x 2}-u_{p y 0}^{\Sigma} \Delta M_{y 2}-u_{p x 0}^{\Sigma} \Delta M_{d c}\right]
\end{aligned}
$$

Then, $\Delta i_{x}$ can be approximated as:

$$
\Delta i_{x} \approx \frac{-M_{x 0} \Delta u_{p 0}^{\Sigma}}{2\left(s L_{\text {tot }}+R_{\text {tot }}\right)}-\frac{\left(u_{p x 20}^{\Sigma}+2 u_{p 00}^{\Sigma}\right) \Delta M_{x}+u_{p y 20}^{\Sigma} \Delta M_{y}}{4\left(s L_{t o t}+R_{\text {tot }}\right)}
$$

Different from (9) and (10), the small-signal models of PCC voltage can be approximated by ignoring $\Delta i_{y}$ :

$$
\begin{gathered}
\Delta u_{p x}=\frac{\omega L_{s}}{K_{T}} \Delta i_{y}-\left(\frac{R_{s}}{K_{T}}+\frac{s L_{s}}{K_{T}}\right) \Delta i_{x} \\
\Delta u_{p y}=-\frac{\omega L_{s}}{K_{T}} \Delta i_{x}-\left(\frac{R_{s}}{K_{T}}+\frac{s L_{s}}{K_{T}}\right) \Delta i_{y}
\end{gathered}
$$

Substitute (31) and (32) into (11), and PLL dynamics can be accordingly obtained as:

$$
\Delta \theta \approx\left[G_{P L L} \frac{s L_{s} u_{p y 0}+R_{s} u_{p y 0}-\omega L_{s} u_{p x 0}}{K_{T}\left(u_{p x 0}^{2}+u_{p x 0}^{2}\right)}\right]_{G_{\theta_{-i x}}} \Delta i_{x}
$$

The ACC $q$-channel output $\Delta M_{q}$ is ignored for ACC $d$-channel analysis. Then, $\Delta M_{x}$ and $\Delta M_{y}$ can be approximated:

$$
\begin{aligned}
& \Delta M_{x}=\cos \theta_{0} \Delta M_{d}-\sin \theta_{0} \Delta M_{q}+G_{M x_{-} \theta} \Delta \theta \\
& \Delta M_{y}=\sin \theta_{0} \Delta M_{d}+\cos \theta_{0} \Delta M_{q}+G_{M y_{-} \theta} \Delta \theta
\end{aligned}
$$

Substituting (34) and (35) into (28), we can get:

$$
\begin{aligned}
\Delta u_{p 0}^{\Sigma} & =\left(\frac{M_{x 0}}{8 C_{a r m} s}\right)_{G_{u c 0_{-} i x}} \Delta i_{x}+\left(\frac{i_{x 0} G_{M x_{-} \theta}+i_{y 0} G_{M_{-} \theta}}{8 C_{a r m} s}\right)_{G_{u c 0_{-} \theta}} \Delta \theta \\
& +\left(\frac{i_{x 0} \cos \theta_{0}+i_{y 0} \sin \theta_{0}}{8 C_{a r m} s}\right)_{G_{u c 0_{-} M d}} \Delta M_{d}
\end{aligned}
$$

Substituting (34) and (35) into (30), we can get:

$$
\begin{aligned}
\Delta i_{x}= & {\left[\frac{-\left(u_{p x 20}^{\Sigma}+2 u_{p 00}^{\Sigma}\right) \cos \theta_{0}-u_{p y 20}^{\Sigma} \sin \theta_{0}}{4\left(s L_{t o t}+R_{t o t}\right)}\right]_{G_{i x_{-} M d}} \Delta M_{d}+} \\
& {\left[\frac{-\left(u_{p x 20}^{\Sigma}+2 u_{p 00}^{\Sigma}\right) G_{M x_{-} \theta}-u_{p y 20}^{\Sigma} G_{M y_{-} \theta}}{4\left(s L_{t o t}+R_{t o t}\right)}\right]_{G_{i x_{-} \theta}} \Delta \theta+} \\
& {\left[\frac{-M_{x 0}}{2\left(s L_{t o t}+R_{t o t}\right)}\right]_{G_{i x-u c 0}} \Delta u_{p 0}^{\Sigma} }
\end{aligned}
$$

For the three equations (33), (36) and (37), consider $\Delta \theta, \Delta i_{x}$ and $\Delta u_{p 0}^{\Sigma}$ as the three unknown variables and $\Delta M_{d}$ as the input, then we can express $\Delta u_{p 0}^{\Sigma}$ and $\Delta i_{x}$ with $\Delta M_{d}$ as:

$$
\Delta u_{p 0}^{\Sigma}=\left\{\begin{array}{l}
\frac{1}{G_{f}}\left[\left(G_{u c 0_{-} i x}+G_{u c 0_{-} \theta} G_{\theta_{-} i x}\right) G_{i x_{-} M d}+\right. \\
\left.G_{u c 0_{-} M d}\left(1-G_{i x_{-} \theta} G_{\theta_{-} i x}\right)\right]
\end{array}\right\}_{G_{u c 0_{-} M d f}} \Delta M_{d}
$$




$$
\Delta i_{x}=\left(\frac{G_{i x_{\_} M d}+G_{i x_{-} u c 0} G_{u c 0_{\_} M d}}{G_{f}}\right)_{G_{i x_{-} M d f}} \Delta M_{d}
$$

where $G_{f}=1-G_{i x_{-} \theta} G_{\theta_{-} i x}-\left(G_{u c 0_{-} i x}+G_{u c 0_{-} \theta} G_{\theta_{-} i x}\right) G_{i x_{-} u c 0}$.

In the following, we should express $\Delta M_{d}$ with $\Delta u_{p 0}^{\Sigma}$ and $\Delta i_{x}$ by considering ACC $d$-channel dynamics.

The complete expression for $\Delta M_{d}$ is:

$$
\begin{gathered}
\Delta M_{d}=G_{i n} G_{\text {fiac }} \Delta i_{d}+L_{p u} G_{\text {fiac }} \Delta i_{q}+G_{\text {fuac }} \Delta u_{p d}- \\
G_{\text {in }} G_{\text {out }}\left(\Delta u_{\text {cavgref }}-G_{\text {fuc }} \Delta u_{p 0}^{\Sigma}\right)
\end{gathered}
$$

Though the participation factors of the signal filters for the $\mathrm{AC}$ current or $\mathrm{AC}$ voltage are little, we retain $\Delta i_{d}, \Delta i_{q}$ and $\Delta u_{p d}$ in (40) since they can be easily expressed with $\Delta i_{x} . \Delta i_{d}$ and $\Delta i_{q}$ can be approximated by ignoring $\Delta i_{y}$ as:

$$
\begin{gathered}
\Delta i_{d}=\cos \theta_{0} \Delta i_{x}+\sin \theta_{0} \Delta i_{y}+ \\
\quad\left(-i_{x 0} \sin \theta_{0}+i_{y 0} \cos \theta_{0}\right)_{G_{i d_{-} \theta}} \Delta \theta \\
\Delta i_{q}=\cos \theta_{0} \Delta i_{y}-\sin \theta_{0} \Delta i_{x}+G_{i q_{-} \theta} \Delta \theta
\end{gathered}
$$

Substituting (33) into (41) and (42), and we can get:

$$
\begin{aligned}
& \Delta i_{d} \approx\left(\cos \theta_{0}+G_{i d_{-} \theta} G_{\theta_{-} i x}\right)_{G_{i d_{-} i x}} \Delta i_{x} \\
& \Delta i_{q} \approx\left(-\sin \theta_{0}+G_{i q_{-} \theta} G_{\theta_{-} i x}\right)_{G_{i q_{-} i x}} \Delta i_{x}
\end{aligned}
$$

$\Delta u_{p d}$ is originally expressed as:

$$
\begin{aligned}
\Delta u_{p d} & =\cos \theta_{0} \Delta u_{p x}+\sin \theta_{0} \Delta u_{p y}+ \\
& \left(-u_{p x 0} \sin \theta_{0}+u_{p y 0} \cos \theta_{0}\right)_{G_{u d_{-} \theta}} \Delta \theta
\end{aligned}
$$

Substituting (31) and (32) into (45), $\Delta u_{p d}$ is represented as:

$$
\begin{aligned}
\Delta u_{p d}= & {\left[G_{u d_{-} \theta} G_{\theta_{-} i x}-\right.} \\
& \left.\frac{L_{s} \cos \theta_{0} s+R_{s} \cos \theta_{0}+\omega L_{s} \sin \theta_{0}}{K_{T}}\right]_{G_{u d_{-} i x}} \Delta i_{x}
\end{aligned}
$$

Then $\Delta M_{d}$ can be expressed as:

$$
\begin{aligned}
& \Delta M_{d}=-G_{i n} G_{\text {out }}\left(\Delta u_{\text {cavgref }}-\Delta u_{\text {cavgfil }}\right)+ \\
& \left(G_{i n} G_{\text {fiac }} G_{i d_{-} i x}+L_{p u} G_{f i a c} G_{i q_{-} i x}+G_{f u a c} G_{u d_{-} i x}\right)_{G_{M d_{-} i x}} \Delta i_{x}
\end{aligned}
$$

The block diagram for ACC $d$-channel control model can be obtained, as shown in Fig. 17. The open-loop transfer function can be obtained as:

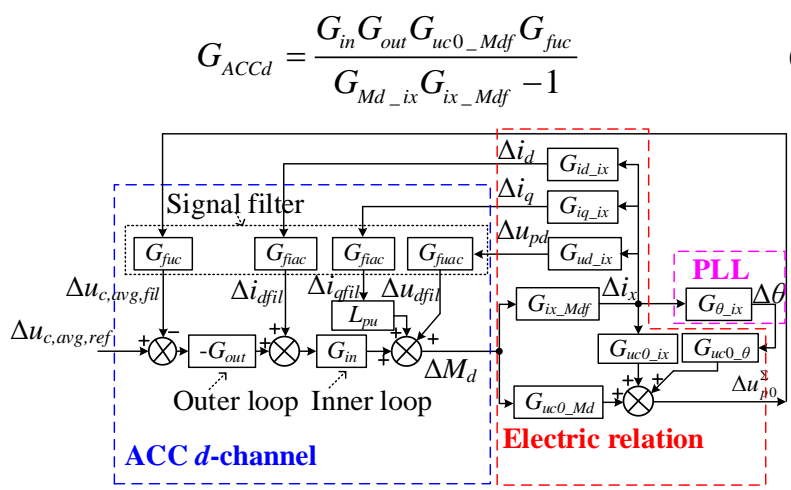

Fig. 17. Transfer function diagram of ACC $d$-channel control for analyzing the instability.

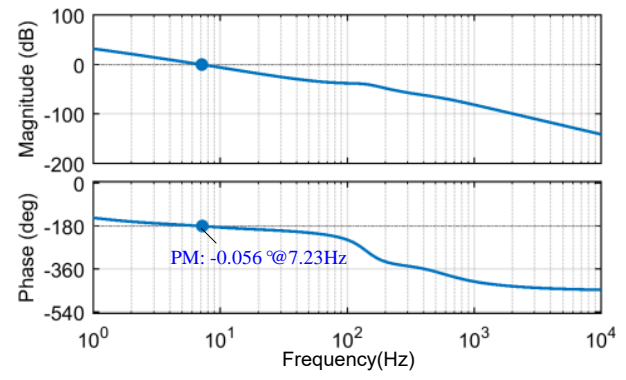

Fig. 18. Bode diagram of $G_{A C C d}$ under $57.50 \%$ rectified active power.

\section{E. Accuracy verification and analysis of $G_{A C C d}(s)$}

Let the rectified active power of the hybrid MMC be the critical value 0.5750 p.u., and the corresponding bode diagram of $G_{A C C d}$ is presented in Fig. 18. It can be seen that the PM of $G_{A C C d}$ is narrowly negative, which indicates a critically unstable state. The crossover frequency is $7.23 \mathrm{~Hz}$, which accords with the mode calculation result $7.22 \mathrm{~Hz}$. Hence, $G_{A C C d}$ is proved to be effective in judging stability and the oscillation frequency for the rectified hybrid MMC connected with the weak AC system. The maximum rectified active power can also be efficiently determined by $G_{A C C d}$.

Similarly, $G_{A C C d}$ can be employed to analyze the impact of control parameters on stability by comparing PMs under different configurations. TABLE II displays the PM variations against different parameters. PM can be obviously increased by raising $K_{P O}$ or reducing $K_{I O}$ for ACC outer loop. In contrast, the increments of PM are not notable for other parameters, such as increasing absorbed reactive power or PLL gain.

To highlight the effective parameter adjustment methods, Fig. 19 depicts the corresponding bode plots. As shown in Fig. 19 (a), when $K_{P O}$ rises from default 0.4 to 1.4 , PM rises to $8.37^{\circ}$. The impact of increasing $K_{P O}$ accords with the sensitivity analysis results in Fig. 10 (b). In Fig. 19 (b), when $K_{I O}$ drops from default 300 to 50 , PM rises to $19.7^{\circ}$. It should be noticed that the increase of $K_{P O}$ or the reduction of $K_{I O}$ results in the rise of control bandwidth of the outer loop for ACC $d$-channel, leading to the acceleration of control effect for the average capacitor voltage.

Based on the analysis above, it is recommended to speed up the ACC $d$-channel control to enhance stability.

TABLE II

PHASE MARgin VARIATION AGAINST DiFFERENT PARAMETERS UNDER RECTIFIER OPERATION

\begin{tabular}{llll}
\hline \hline Parameters & & Value variation & Phase margin variation \\
\hline \multirow{4}{*}{$\mathrm{ACC}$} & $K_{P O}$ & $0.4 \rightarrow 1.4$ & $-0.056^{\circ} \rightarrow 8.37^{\circ}$ \\
& $K_{I O}$ & $300 \rightarrow 50$ & $-0.056^{\circ} \rightarrow 19.7^{\circ}$ \\
& $K_{P I}$ & $1 \rightarrow 2$ & $-0.056^{\circ} \rightarrow 0.92^{\circ}$ \\
& $K_{I I}$ & $50 \rightarrow 500$ & $-0.056^{\circ} \rightarrow 0.68^{\circ}$ \\
\hline \multirow{3}{*}{ Signal filters } & $f_{\text {uac }}$ & $300 \mathrm{~Hz} \rightarrow 50 \mathrm{~Hz}$ & $-0.056^{\circ} \rightarrow 0.82^{\circ}$ \\
& $f_{\text {iac }}$ & $800 \mathrm{~Hz} \rightarrow 300 \mathrm{~Hz}$ & $-0.056^{\circ} \rightarrow 1.17^{\circ}$ \\
& $f_{u c}$ & $250 \mathrm{~Hz} \rightarrow 1000 \mathrm{~Hz}$ & $-0.056^{\circ} \rightarrow 0.53^{\circ}$ \\
\hline PLL & $K_{P p l l}$ & $80 \rightarrow 120$ & $-0.056^{\circ} \rightarrow 1.83^{\circ}$ \\
\hline Reactive power & $Q$ & $-10 \% \rightarrow 20 \%$ (p.u.) & $-0.056^{\circ} \rightarrow 0.863^{\circ}$ \\
\hline \hline
\end{tabular}




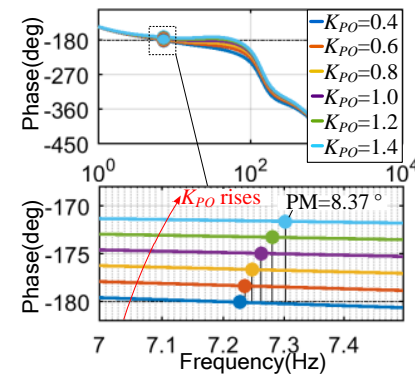

(a) increasing $K_{P O}$

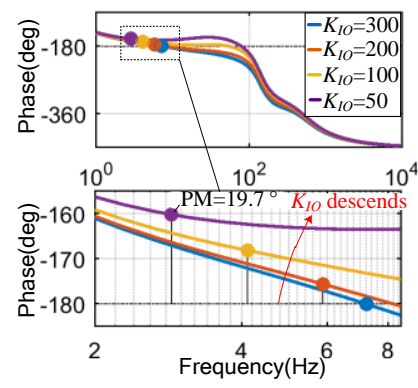

(b) decreasing $K_{I O}$
Fig. 19. Effective parameter adjustment methods to increase phase margins.

\section{V.OSCILLATION MiTIGATION VERIFICATION}

In this section, the effectiveness of the parameter adjustment on damping the oscillation caused is validated by electromagnetic simulation. The enhancement of the transferable active power is also verified. A detailed calculation of the parameter adjustment versus the maximum stability-limited active power can be referred to [30]. It should be noticed that when the system configuration is varied, for example, the AC system SCR or MMC arm reactance are changed, we can similarly re-calculate the relationship and obtain suitable adjustment ranges for the control parameters by the small-signal models in this paper.

As shown in Fig. 20 (a), the inverted active power of the master hybrid MMC reaches 0.6 p.u. at $1.75 \mathrm{~s}$, and the reactive power starts to oscillate. At $1.8 \mathrm{~s}, K_{P I}$ is reduced from 1 to 0.5 , and the oscillation is suppressed effectively, then the inverted active power continues rising up to 0.9 p.u. with no oscillation. Similarly, in Fig. 20 (b), the high-frequency oscillation is successfully suppressed after $f_{\text {uac }}$ is reduced from $300 \mathrm{~Hz}$ to $100 \mathrm{~Hz}$, and the inverted active power continues rising up to 1 p.u..

As shown in Fig. 20 (c), the rectified active power of the master hybrid MMC reaches 0.65 p.u. at $3.75 \mathrm{~s}$, and the average capacitor voltage starts to oscillate. At $5.5 \mathrm{~s}, K_{P O}$ is increased from 0.4 to 1.4 , and the oscillation is suppressed, then the active power continues to rise up to 0.9 p.u.. Similarly in Fig. 20 (d), the low-frequency oscillation is effectively suppressed after $K_{I O}$ is reduced from 300 to 10 , and the rectified active power continues to rise up to 0.9 p.u. with retaining stability, but the oscillation is poorly damped.

According to Fig. 16 (c), decreasing $f_{p}$ is supposed to be effective in improving stability. While the step response of ACC $q$-channel inner-loop is shown in Fig. 21 (a), indicating that this method may increase the overshoot of $i_{q f i}$. Moreover, PLL gain is recommended to be reduced under weak AC system integration by many researchers such as in [9],[11],[13] for two-level VSCs, which is also proved to be effective in damping the oscillation in this paper, however, it introduces serious deterioration towards the dynamic performance of ACC $q$-channel outer-loop control, as shown in Fig. 21 (b). This is also proved in bode analysis for $G_{A C C q}$, as shown in Fig. 22 (a). When $K_{P p l l}$ is much reduced, though the stability margin of $G_{A C C q}$ in the high-frequency range is slightly improved, the stability margin in the low-frequency range is worsened, indicating underdamped low frequency oscillation, as reflected in Fig. 21 (b). While for the rectifier operation, as shown in Fig. 22 (b), the increase of $K_{P p l l}$ leads to slight stability improvement in the low-frequency oscillation range.

The final suggested parameter adjustment methods are given in TABLE III. It should be noted that when multiple parameters are adjusted, TABLE III can be used to direct the adjustment.

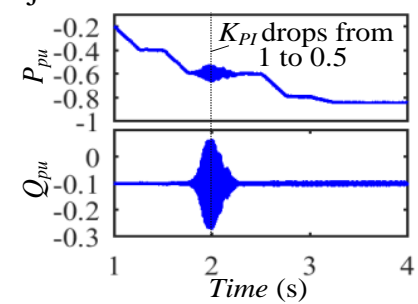

(a) decreasing $K_{P I}$

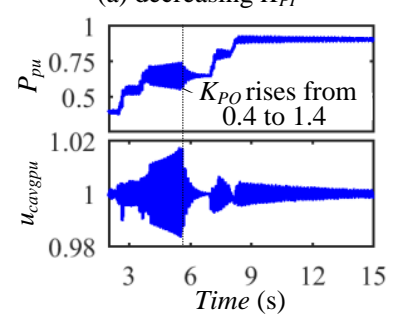

(c) increasing $K_{P O}$

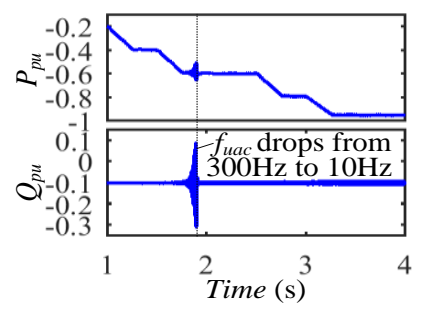

(b) decreasing $f_{\text {uac }}$

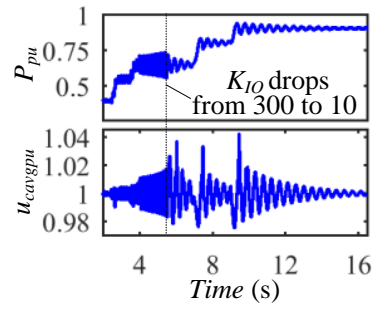

(d) decreasing $K_{I O}$
Fig. 20. Effectiveness of different parameters adjustment on suppressing oscillation and enhancing transferable active power.

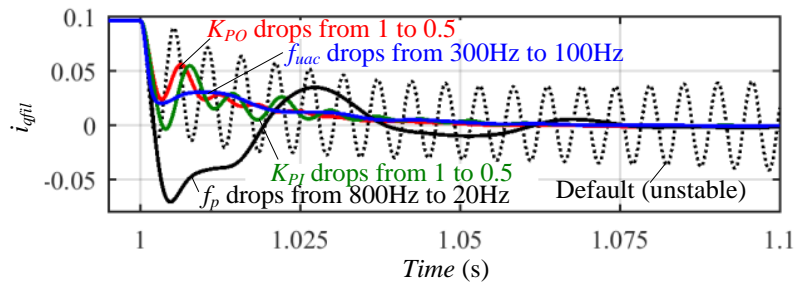

(a) inverter status

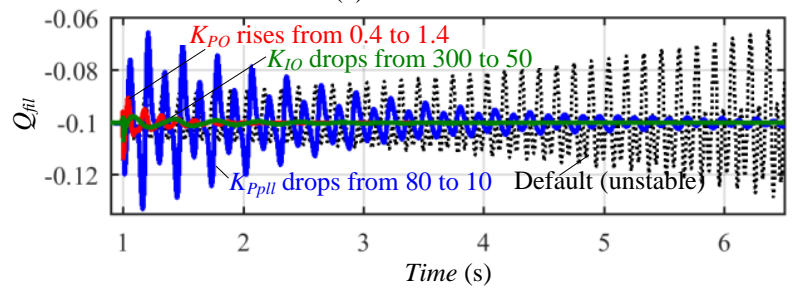

(b) rectifier status

Fig. 21. Dynamic performance of parameter adjustment methods.

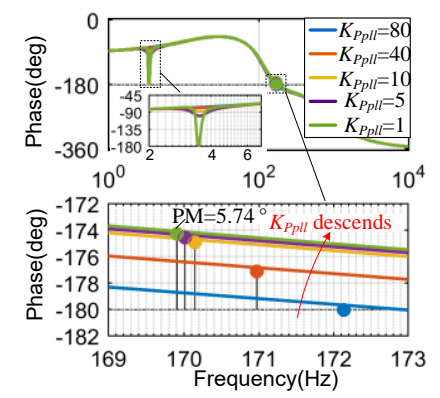

(a) $G_{A C C q}$ for inverter operation

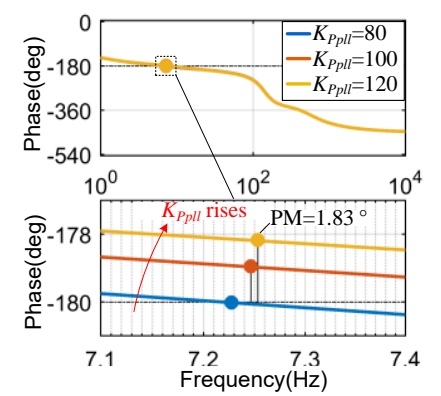

(b) $G_{A C C d}$ for rectifier operation Fig. 22. Varying PLL gain $K_{P p l l}$ to increase phase margins under different operation states.

TABLE III

RECOMMENDED PARAMETER ADJUSTMENT METHOD

\begin{tabular}{ccc}
\hline $\begin{array}{c}\text { Operation } \\
\text { status }\end{array}$ & $\begin{array}{c}\text { Suggested parameter } \\
\text { adjustment }\end{array}$ & Physical explanation \\
\cline { 1 - 1 } inverter & Decrease $K_{P O}$ or $K_{P I}$ & Decelerate the outer or inner loop of \\
\cline { 1 - 1 } &
\end{tabular}



subject to Institution of Electrical and Electronic Engineering Copyright. The copy of record is available at IEEE Xplore.

\begin{tabular}{lcc}
\hline & ACC $q$-channel control \\
& Decrease $f_{\text {uac }}$ & $\begin{array}{c}\text { Decrease bandwidth of the low-pass } \\
\text { signal filter for PCC voltage }\end{array}$ \\
\hline \multirow{2}{*}{ rectifier } & $\begin{array}{c}\text { Increase } K_{P O}, \\
\text { decrease } K_{I O}\end{array}$ & $\begin{array}{c}\text { Accelerate the outer loop of ACC } \\
\end{array}$ \\
\hline
\end{tabular}

\section{CONCLUSIONS}

This paper investigates a wide-band oscillation of the master hybrid MMC connected to the weak AC grid triggered by excessive penetration of the active power. A high-frequency (several hundred $\mathrm{Hz}$ ) and low-frequency (several $\mathrm{Hz}$ ) oscillations can be activated under the inverter and rectifier status separately.

The full-order time-domain small-signal model is developed. Key parameters and dominant state variables regarding the unstable modes are identified through sensitivity and participation factor analysis. The oscillation is considered to be triggered by the mismatch of control speed towards the weak AC grid condition. The high-frequency oscillation is related to over-quick ACC $q$-channel control, while the low-frequency oscillation is related to over-slow ACC $d$-channel control.

Simplified s-domain models to calculate the closed-loop control gain for ACC $d / q$-channel are developed referring to SMA approach. Sensitivity analysis towards phase margins reveals effective parameter adjustment methods that can enhance the stability. Generally, when the hybrid MMC is connected to a weak AC grid or facing a sudden SCR dip of the $\mathrm{AC}$ system, the proportion gain of the ACC outer and inner loop, or the cutting-off frequency for PCC voltage filter are recommended to be relatively small under inverter status; while under rectifier status, the proportion /integral gain of the ACC outer loop is suggested to be increased/decreased.

\section{APPENDIX}

TABLE A I

PARAMETERS OF THE TESTED HYBRID MMC AND AC SYSTEM

\begin{tabular}{lll}
\hline \hline & \multicolumn{1}{c}{ Parameters } & \multicolumn{1}{c}{ Values } \\
\hline & Rated DC voltage & $640 \mathrm{kV}$ \\
& Rated capacity & $1000 \mathrm{MVA}$ \\
Hybrid & Arm inductance & $112 \mathrm{mH}$ \\
MMC & Arm equivalent resistance & $0.01 \Omega$ \\
& Number of FBSM/HBSM & $100 / 100$ \\
& Submodule capacitance & $10 \mathrm{mF}$ \\
\hline AC & Rated voltage grid/converter-side & $400 \mathrm{kV} / 352 \mathrm{kV}$ \\
transformer & Leak inductance & $15 \%$ \\
\hline \multirow{3}{*}{ AC system } & Rated voltage & $400 \mathrm{kV}$ \\
& Internal impedance (AC system 1$)$ & $80 \angle 85.62^{\circ} \Omega$ \\
& Internal impedance (AC system 2$)$ & $8 \angle 82.83^{\circ} \Omega$ \\
\hline \multirow{4}{*}{ DC line } & $L_{d c}$ & $100 \mathrm{mH}$ \\
& $C_{\text {line }}$ & $2 \mu \mathrm{F}$ \\
& $L_{\text {line }}$ & $1 \mathrm{mH}$ \\
\hline \hline
\end{tabular}

TABLE A II

CONTROL PARAMETERS OF THE HYBRID MMC1

\begin{tabular}{ccc}
\hline \hline \multicolumn{2}{c}{ Control parameters } & Values \\
\hline \multirow{2}{*}{ PLL } & $K_{\text {Ppll }}$ & 80 \\
& $K_{\text {Ipll }}$ & 500 \\
\hline \multirow{3}{*}{ Signal } & $f_{p}$ & $800 \mathrm{~Hz}$ \\
filters & $f_{\text {uac }}$ & $300 \mathrm{~Hz}$ \\
& $f_{\text {iac }}$ & $800 \mathrm{~Hz}$ \\
& $f_{\text {udc }}$ & $300 \mathrm{~Hz}$
\end{tabular}

\begin{tabular}{ccc}
\hline & $f_{\text {idc }}$ & $800 \mathrm{~Hz}$ \\
& $f_{u c}$ & $500 \mathrm{~Hz}$ \\
\hline \multirow{4}{*}{$\mathrm{ACC}$} & $K_{P O}$ & 1 (Inverter) 0.4 (Rectifier) \\
& $K_{I O}$ & 100 (Inverter) 300 (Rectifier) \\
& $K_{P I}$ & 1 \\
& $K_{I I}$ & 50 \\
\hline \multirow{4}{*}{ DCC } & $K_{P O d c}$ & 1.2 \\
& $K_{I O d c}$ & 800 \\
& $K_{P I d c}$ & 2 \\
& $K_{I d c}$ & 100 \\
\hline \hline
\end{tabular}

\section{REFERENCES}

[1] W. Xiang, S. Yang, L. Xu, J. Zhang, W. Lin and J. Wen, "A Transient Voltage-Based DC Fault Line Protection Scheme for MMC-Based DC Grid Embedding DC Breakers," IEEE Trans. Power Del., vol. 34, no. 1, pp. 334-345, Feb. 2019.

[2] B. Li, J. He, Y. Li, B. Li. "A review of the protection for the multi-terminal VSC-HVDC grid". Prot. Control Mod. Power Syst., vol.4, no. 4, pp:239-249, Nov. 2019.

[3] H. Rao, Y. Zhou, S. Xu, et al., "Key technologies of ultra-high voltage hybrid LCC-VSC MTDC systems," CSEE J. Power Energy Syst., vol. 5, no. 3, pp. 365-373, Sept. 2019.

[4] W. Lin, D. Jovcic, S. Nguefeu and H. Saad, "Full-Bridge MMC Converter Optimal Design to HVDC Operational Requirements," IEEE Trans. Power Del., vol. 31, no. 3, pp. 1342-1350, June 2016.

[5] W. Xiang, W. Lin, L. Xu and J. Wen, "Enhanced Independent Pole Control of Hybrid MMC-HVdc System," IEEE Trans. Power Del., vol. 33, no. 2, pp. 861-872, April 2018.

[6] Y. Li et al., "Power Compensation Control for Interconnection of Weak Power Systems by VSC-HVDC," IEEE Trans. Power Del., vol. 32, no. 4, pp. 1964-1974, Aug. 2017.

[7] C. Yin, X. Xie, S. Xu and C. Zou, "Review of oscillations in VSC-HVDC systems caused by control interactions," J. Eng., vol. 2019, no. 16, pp. 1204-1207, March 2019.

[8] C. Zou, H. Rao, S. Xu, et al. "Analysis of Resonance Between a VSC-HVDC Converter and the AC Grid," IEEE Trans. Power Electron., vol. 33, no. 12, pp. 10157-10168, Dec. 2018.

[9] J. Z. Zhou, H. Ding, S. Fan, Y. Zhang and A. M. Gole, "Impact of Short-Circuit Ratio and Phase-Locked-Loop Parameters on the Small-Signal Behavior of a VSC-HVDC Converter," IEEE Trans. Power Del., vol. 29, no. 5, pp. 2287-2296, Oct. 2014.

[10] A. Egea-Alvarez, S. Fekriasl, F. Hassan and O. Gomis-Bellmunt, "Advanced Vector Control for Voltage Source Converters Connected to Weak Grids," IEEE Trans. Power Syst., vol. 30, no. 6, pp. 3072-3081, Nov. 2015.

[11] M. F. M. Arani and Y. A. I. Mohamed, "Analysis and Performance Enhancement of Vector-Controlled VSC in HVDC Links Connected to Very Weak Grids," IEEE Trans. Power Syst., vol. 32, no. 1, pp. 684-693, Jan. 2017.

[12] B. Wen, D. Boroyevich, R. Burgos, P. Mattavelli and Z. Shen, "Analysis of D-Q Small-Signal Impedance of Grid-Tied Inverters," IEEE Trans. Power Electron., vol. 31, no. 1, pp. 675-687, Jan. 2016.

[13] B. Wen, D. Dong, D. Boroyevich, R. Burgos, P. Mattavelli and Z. Shen, "Impedance-Based Analysis of Grid-Synchronization Stability for Three-Phase Paralleled Converters," IEEE Trans. Power Electron., vol. 31, no. 1, pp. 26-38, Jan. 2016.

[14] S. Sang, N. Gao, X. Cai and R. Li, "A Novel Power-Voltage Control Strategy for the Grid-Tied Inverter to Raise the Rated Power Injection Level in a Weak Grid," IEEE J. Emerg. Sel. Top. Power Electron., vol. 6, no. 1, pp. 219-232, March 2018.

[15] Y. Huang, X. Yuan, J. Hu, P. Zhou and D. Wang, "DC-Bus Voltage Control Stability Affected by AC-Bus Voltage Control in VSCs Connected to Weak AC Grids," IEEE J. Emerg. Sel. Top. Power Electron., vol. 4, no. 2, pp. 445-458, June 2016.

[16] D. Wang, L. Liang, L. Shi, J. Hu and Y. Hou, "Analysis of Modal Resonance Between PLL and DC-Link Voltage Control in Weak-Grid Tied VSCs," IEEE Trans. Power Syst., vol. 34, no. 2, pp. 1127-1138, March 2019.

[17] J. Khazaei, M. Beza and M. Bongiorno, "Impedance Analysis of Modular Multi-Level Converters Connected to Weak AC Grids," IEEE Trans. Power Syst., vol. 33, no. 4, pp. 4015-4025, July 2018. 
[18] A. Jamshidifar and D. Jovcic, "Small-Signal Dynamic DQ Model of Modular Multilevel Converter for System Studies," IEEE Trans. Power Del., vol. 31, no. 1, pp. 191-199, Feb. 2016.

[19] M. Mehrasa, E. Pouresmaeil, S. Zabihi et al, "Dynamic Model, Control and Stability Analysis of MMC in HVDC Transmission Systems," IEEE Trans. Power Del., vol. 32, no. 3, pp. 1471-1482, June 2017.

[20] J. Freytes, G. Bergna, J. A. Suul, et al, "Improving Small-Signal Stability of an MMC with CCSC by Control of the Internally Stored Energy," IEEE Trans. Power Del., vol. 33, no. 1, pp. 429-439, Feb. 2018.

[21] X. Lu, W. Xiang, W. Lin, J. Wen, "Small-signal modeling of MMC based DC grid and analysis of the impact of DC reactors on the small-signal stability," Int. J. Electr. Power Energy Syst. vol. 101, pp. 25-37, October 2018.

[22] M. Amin, M. Molinas, J. Lyu and X. Cai, "Impact of Power Flow Direction on the Stability of VSC-HVDC Seen from the Impedance Nyquist Plot," IEEE Trans. Power Electron., vol. 32, no. 10, pp. 8204-8217, Oct. 2017.

[23] I. J. Perez-arriaga, G. C. Verghese and F. C. Schweppe, "Selective Modal Analysis with Applications to Electric Power Systems, PART I: Heuristic Introduction," IEEE Trans. Power Appar. Syst., vol. PAS-101, no. 9, pp. 3117-3125, Sept. 1982.

[24] A. Beghi, "An application of selective modal analysis to tokamak modeling and control," IEEE Trans. Control Syst. Technol., vol. 9, no. 4, pp. 574-589, July 2001.

[25] A. Cenedese, M. Fagherazzi and P. Bettini, "A Novel Application of Selective Modal Analysis to Large-Scale Electromagnetic Devices," IEEE Trans. Magn., vol. 52, no. 3, pp. 1-4, March 2016.

[26] X. Lu, W. Xiang, W. Lin, J. Wen. "State-space model and PQ operation zone analysis of hybrid MMC," Electr. Power Syst. Res., vol. 162, pp. 99-108, September 2018.

[27] N. Trinh, M. Zeller, K. Wuerflinger and I. Erlich, "Generic Model of MMC-VSC-HVDC for Interaction Study with AC Power System," IEEE Trans. Power Syst., vol. 31, no. 1, pp. 27-34, Jan. 2016.

[28] S. Arunprasanth, U. D. Annakkage, C. Karawita and R. Kuffel, "Generalized Frequency-Domain Controller Tuning Procedure for VSC Systems," IEEE Trans. Power Del., vol. 31, no. 2, pp. 732-742, April 2016.

[29] G. Pinares and M. Bongiorno, "Modeling and Analysis of VSC-Based HVDC Systems for DC Network Stability Studies," IEEE Trans. Power Del., vol. 31, no. 2, pp. 848-856, April 2016.

[30] X. Lu, W. Xiang and J. Wen, "Enhancing Active Power Transfer Capability for Hybrid MMC Integrated with Weak AC Grid through Parameter Adjustment," Proc. 45th Annu. Conf. IEEE Ind. Electron. Soc., Lisbon, Portugal, 2019, pp. 4805-4810.

[31] P. Kundur. Power system stability and control. McGraw-Hill, 1994.

\section{BIOGRAPHIES}

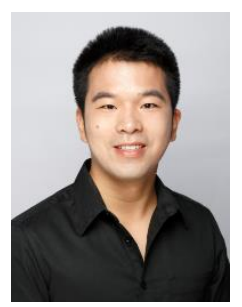

Xiaojun Lu received his B.Eng. and $\mathrm{PhD}$ degrees both in electrical engineering from Huazhong University of Science and Technology (HUST), China in 2013 and 2018, respectively. Currently, he is a postdoctor with HUST since 2018. His main research interests include modeling and stability analysis of MMC-HVDC and dc grids.

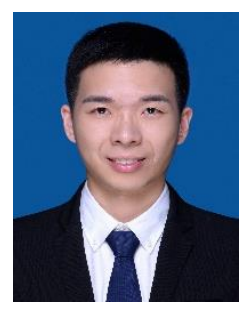

Wang Xiang (S'16-M'17) received his B.Eng. and $\mathrm{PhD}$ degrees both in electrical engineering from Huazhong University of Science and Technology (HUST), China in 2012 and 2017, respectively. He was a visiting student at the University of Aberdeen and the University of Strathclyde in 2014 and 2016 respectively. Currently, he is a research associate with the University of Strathclyde since 2018. His main research interests include MMC-HVDC, high power $\mathrm{dc} / \mathrm{dc}$ converters and dc grids.

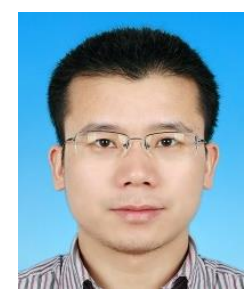

Weixing Lin (M'13) obtained his B.E. and $\mathrm{PhD}$ degrees in electrical engineering from Huazhong University of Science and Technology (HUST), China, in 2008 and 2014 respectively. He was a research fellow at University of Aberdeen during 2012-2016. $\mathrm{He}$ is currently the chief engineer of HVDC division at TBEA China Xinjiang Sunoasis Co. Ltd. His research interests are HVDC, MMC, dc-dc autotransformer, DC grids.

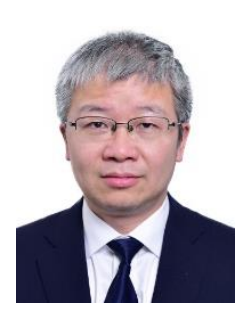

Jinyu Wen (M'10) received his B.Eng. and Ph.D. degrees all in electrical engineering from Huazhong University of Science and Technology (HUST), Wuhan, China, in 1992 and 1998, respectively. He was a visiting student from 1996 to 1997 and a research fellow from 2002 to 2003 all at the University of Liverpool, UK, and a senior visiting researcher at the University of Texas at Arlington, USA in 2010. From 1998 to 2002 he was a director engineer in XJ Electric Co. Ltd. in China. In 2003 he joined the HUST and now is a Professor at HUST. His current research interests include renewable energy integration, energy storage application, DC grid, and power system operation and control. 